\title{
SMOOTH LINEARIZATION FOR A SADDLE ON BANACH SPACES.
}

\author{
HILDEBRANDO M. RODRIGUES ${ }^{\dagger}$ AND J. SOLÀ-MORALES $\ddagger$
}

\begin{abstract}
Dedicated to Prof. Shui-Nee Chow, on occasion of his sixtieth birthday.
Abstract. As a continuation of a previous work on linearization of class $C^{1}$ of diffeomorphisms and flows in infinite dimensions near a fixed point, in this work we deal with the case of a saddle point with some non-resonance restrictions for the linear part. Our result can be seen as an extension of results by P. Hartman [2] and Aronson, Belitskii and Zhuzhoma [1] in dimension two. We also present an application to a system of nonlinear wave equations.
\end{abstract}

Keywords: linearization, conjugacy, wave equation.

AMS Classification. Primary: 35B05, 34G20. Secondary: 35B40, 34D05.

\section{Introduction AND MAin Result.}

Local linearization near an equilibrium point of a dynamical system, either discrete or continuous, is an important and widely used tool to analyze local dynamics and asymptotic behavior of orbits. As a mathematical topic it has a very long history, and in many aspects it is still a subject of contemporary research. The reader can see in Rodrigues\&Solà-Morales[5] a short account of these facts.

In the case of discrete dynamical systems, the problem of local linearization is the following: one has a smooth nonlinear map $T$ from a linear space $\mathbb{X}$ into itself such that $T(0)=0$ and

Date: October 30, 2003.

$\dagger$ Departamento de Matemática, Instituto de Ciências Matemáticas e de Computação, Universidade de São Paulo, Caixa Postal 668, 13560-970, São Carlos, SP, Brazil,

e-mail: hmr@icmc.sc.usp.br, phone: (55)(16)273-9632.

$\ddagger$ Departament de Matemàtica Aplicada 1, Universitat Politècnica de Catalunya, Av. Diagonal 647, 08028 Barcelona, Spain, e-mail: jc.sola-morales@upc.es.

$\dagger$ Partially supported by Fapesp, CNPq and CAPES/MECD 023/01, Brazil.

$\ddagger$ Partially supported by Fapesp, Brazil, and MECD, Spain (BFM2000-0962-C02-02 and PHB2001-0052PC, BMF2002-04613-C03-01) . 
asks oneself for the existence of an invertible map $R: \mathbb{X} \rightarrow \mathbb{X}$ with $R(0)=0$ such that $R T R^{-1} x=L x$ for all $x$ in some neighborhood of 0 in $\mathbb{X}$, where $L: \mathbb{X} \rightarrow \mathbb{X}$ is linear, usually $L=D T(0)$. We restrict our considerations to the case where $T$ is invertible, at least locally.

The most classical result, the so called Hartman-Grobman Theorem deals with the case where $R$ is asked to be merely continuous, and it is known to hold even when $\mathbb{X}$ is Banach space of infinite dimension. There are also other very important results in which $R$ is asked to be smoother, but only a few of them Mora\&Solà-Morales[4], Tan[8](Theorem 8.2 an Theorem 8.3), and Rodrigues\&Sola-Morales[5], to our knowledge) are valid when $\mathbb{X}$ is an infinite dimensional Banach space.

There are several reasons to support the interest of $C^{1}$ linearization results in comparison with those in the class $C^{0}$. As it is said in van Strien[9], with a $C^{0}$ linearization it is not possible even to distinguish if the fixed point is a node or a focus. Another reason is that when one deals with a continuous dynamical system coming from a differential equation one also can ask if the strict solutions of the differential equation are transformed by $R$ to strict solutions of a new differential equation, and this needs differentiability. A third reason is the relevance of the invariant manifolds of class $C^{1}$ that one would like to keep also preserved by $R$.

Concerning linearization in $\mathbb{R}^{n}$ in the class $C^{m}$ with $m>0$ it is known that necessary non-resonance conditions in the form of diophantine inequalities have to be satisfied by the eigenvalues of the linear part (see Sell[6], for example). But it was pointed out in Hartman[2] that there are two cases where these diophantine inequalities need not to be assumed, both cases with $m=1$, namely the case where $T$ is a local contraction and the case where $n=2$ and $T$ is hyperbolic.

In the present paper, we have been looking to possible extensions to infinite dimensional Banach spaces of the results for smooth linearization that are already known in finite dimensions. Our original motivation was the partial result given in Mora\&Solà-Morales[4]. And we have started with the two cases mentioned, where no diophantine inequalities are needed. Roughly speaking, our approach has consisted in substituting the role of the eigenvalues 
of $D T(0)$ in the finite dimensional theorems for blocks, or spectral sets of the spectrum $\sigma(D T(0))$ of the form $\{\lambda \in \sigma(D T(0))|a<| \lambda \mid<b\}$.

Our first result dealt with the case of contractions, and it is the object of our previous paper Rodrigues\&Solà-Morales[5]. Its main result is the following theorem, that contains nonresonance conditions that can be seen as reminiscent from the diophantine inequalities of the finite dimensional cases. But the reader can observe that when this theorem is applied to a finite dimensional situation the numbers $\nu_{i}^{-}, \nu_{i}^{+}$can always be suitably chosen in order to fulfill the nonresonance condition.

Theorem 1. (The Linearization Theorem For Contractions.) Let $\mathbb{X}$ be a Banach space with the property that there exists function $\phi$ such that

$$
\Phi \in C^{1,1}(\mathbb{X}, \mathbb{R}), \text { with } \Phi(x)=1 \text {, when }|x| \leq 1 / 2 \text { and } \Phi(x)=0 \text {, when }|x| \geq 1 \text {. }
$$

Suppose that $A, A^{-1} \in L(\mathbb{X})$. We assume that there exist real numbers $\nu_{i}^{-}, \nu_{i}^{+}$, $i=1, \cdots, n$ such that:

$$
\left.\begin{array}{l}
0<\nu_{n}^{-}<\nu_{n}^{+}<\nu_{n-1}^{-}<\nu_{n-1}^{+}<\cdots \nu_{1}^{-}<\nu_{1}^{+}<1 \\
\nu_{1}^{+} \nu_{i}^{+}<\nu_{i}^{-}, i=1, \cdots, n \quad \text { (nonresonance condition) } \\
|\sigma(A)| \subset\left(\nu_{n}^{-}, \nu_{n}^{+}\right) \cup\left(\nu_{n-1}^{-}, \nu_{n-1}^{+}\right) \cup \cdots \cup\left(\nu_{1}^{-}, \nu_{1}^{+}\right) .
\end{array}\right\}
$$

Let $X=X(x)$ be a $C^{1,1}$-function in a neighborhood of the origin with values in $\mathbb{X}$, such that $X=0, \partial_{x} X=0$, at $x=0$.

Then, for the map $T: x \mapsto x^{1}, x^{1}=A x+X(x)$, there exists a $C^{1}$-map $R: x \mapsto u$, $u=x+\phi(x)$, satisfying $\phi=0, \partial_{x} \phi=0$, at $x=0$, such that $R T R^{-1}: u \mapsto u^{1}$ has the form $u^{1}=A u$ in a sufficiently small neighborhood of the origin.

The next is the main result of the present paper. It deals with the smooth linearization of a nonlinear saddle, when the modulus of the spectrum of the linear part can be divided in two blocks. The conditions on the blocks take the form of a nonresonance condition.

Theorem 2. (The Linearization Theorem for a Saddle.) Let $\mathbb{Z}$ be a Banach space with the property that there exists function $\Phi$ satisfying condition (1.1). 
Suppose that $L, L^{-1} \in L(\mathbb{Z})$. We assume that there exist real numbers $s^{-}, s^{+}, u^{-}, u^{+}$ such that

$$
\left.\begin{array}{l}
0<s^{-}<s^{+}<1<u^{-}<u^{+} \\
s^{+} u^{+}<u^{-}, s^{+}<s^{-} u^{-},\left(s^{+}\right)^{2}<s^{-}, u^{+}<\left(u^{-}\right)^{2} \quad \text { (nonresonance condition) } \\
|\sigma(L)| \subset\left(s^{-}, s^{+}\right) \cup\left(u^{-}, u^{+}\right) .
\end{array}\right\}
$$

Let $Z=Z(z)$ be a $C^{1, \alpha}$-function in a neighborhood of the origin with values in $\mathbb{Z}$, such that $Z=0, \partial_{z} Z=0$, at $z=0$.

If $\alpha$ is sufficiently close to 1 , then for the map $T: z \mapsto z^{1}, z^{1}=L z+Z(z)$, there exists a $C^{1}$-map $R: z \mapsto u, u=z+\phi(z)$, satisfying $\phi=0, \partial_{z} \phi=0$, at $z=0$, such that $R T R^{-1}: u \mapsto u^{1}$ has the form $u^{1}=L u$ in a sufficiently small neighborhood of the origin.

Remark 1. By an argument due to Sternberg [7], if $T(t)=: e^{L t}+S(t)$ is a group of $C^{1, \alpha}$-diffeomorphisms, where $S(t) 0=0,\left.\left(D_{z} S(t)\right)\right|_{z=0}=0$ and $A=: e^{L}, T=: T(1)$, and if the above hypotheses are satisfied and we define $\bar{R}=: \int_{0}^{1} e^{-L s} R T(s) d s$, then one has $\bar{R} T(t) \bar{R}^{-1}=e^{L t}, \forall t \in \mathbb{R}$.

Some sufficient conditions for the groups $T(t)$ be of class $C^{1, \alpha}$ are included in a general result by D. Henry in [3].

This paper is organized as follows. In Section 2 we first state Theorem 3 that establishes a relationship between the spectral radius of a family of commuting operators with their norms and this result will be important to obtain a decomposition of the whole space into subspaces with special properties. To prove some lemmas and theorems, we have to extend our local mappings to the whole space. Therefore, Lemma 1 says that we can do that preserving some Hölder estimations. In this section we also study some special spaces that will be used to obtain our main result. In Section 3 we prove the existence of solutions of certain abstract functional equations and this will be used in Section 4, where we prove our main theorem. In Section 5 we present an application of our results to a system of abstract wave equations, indicating the conditions on some parameters, in order to have the nonresonance conditions fulfilled. 


\section{Preliminaries.}

The following result and its corollary, that will be used in the next section, were stated and proved in [5].

Theorem 3. Let $\mathbb{X}$ be a Banach space with norm $|\cdot|$. We indicate also by $|\cdot|$ the induced norm of bounded operators on $L(\mathbb{X})$. Let us denote by $r(A)$ the spectral radius of $A$. Let $A_{1}, A_{2}, \cdots, A_{p} \in L(\mathbb{X})$, such that $A_{i} A_{j}=A_{j} A_{i}$, for $i, j=1,2, \cdots, p$. Then, given $\varepsilon>0$, there exists a norm, $\|\cdot\|$ on $\mathbb{X}$, equivalent to $|\cdot|$ such that $r\left(A_{i}\right) \leq\left\|A_{i}\right\| \leq r\left(A_{i}\right)+\varepsilon$, for $i=1,2, \cdots, p$.

Corollary 1. Let $\mathbb{X}$ be a Banach space with norm $|\cdot|$. Suppose that $A, A^{-1} \in L(\mathbb{X})$ and that $|\sigma(A)| \subset(c, d)$, with $c>0$. Then there exists an equivalent norm, $\|\cdot\|$ on $\mathbb{X}$, such that we have $\|A\|<d$ and $\left\|A^{-1}\right\|<\frac{1}{c}$.

The decomposition of the spectrum of $L$ as $\{\lambda \in \mathbb{C}:|\lambda|<1\} \cup\{\lambda \in \mathbb{C}:|\lambda|>1\}$ induces a decomposition of the space $\mathbb{Z}$ as $\mathbb{Z}=\mathbb{X} \times \mathbb{Y}$. We write $z=(x, y), Z(x, y)=(X(x, y), Y(x, y))$ and $|z|=|x|+|y|$.

As a preliminary step for the proof of Theorem 2, we extend our local function $Z$ to a function defined on the whole space $\mathbb{Z}$ by writing:

$$
Z_{r}(x, y)=: \Phi(x / r, y / r) Z(x, y)
$$

where $r>0$ is small enough and $\Phi$ is the $\mathcal{C}^{1,1}$ function given by (1.1). Observe that $Z_{r}(x, y)=Z(x, y)$ if $|x|+|y| \leq \frac{r}{2}$ and that $Z_{r}(x, y, z)=0$ if $|x|+|y| \geq r$.

We want to prove Theorem 2 for the global map:

$$
\left\{\begin{array}{l}
x^{1}=L_{s} x+X_{r}(x, y) \\
y^{1}=L_{u} y+Y_{r}(x, y)
\end{array}\right.
$$

where $L=\left(L_{s}, L_{u}\right)$ and $Z_{r}=\left(X_{r}, Y_{r}\right)$.

Lemma 1. If $\Phi$ is the $\mathcal{C}^{1,1}$ function given by (1.1) and $X, Y$ are $\mathcal{C}^{1, \alpha}$ in a neighborhood of the origin then the functions $X_{r}, Y_{r}$, defined above, are $\mathcal{C}^{1, \alpha}$ globally and the quantities:

$$
\sup _{\left(x_{1}, y_{1}\right) \neq\left(x_{2}, y_{2}\right)} \frac{\left.\mid \partial_{(x, y)} X_{r}\left(x_{1}, y_{1}\right)\right)-\partial_{(x, y)} X_{r}\left(x_{2}, y_{2}\right) \mid}{\left(\left|x_{1}-x_{2}\right|+\left|y_{1}-y_{2}\right|\right)^{\alpha}},
$$




$$
\sup _{\left(x_{1}, y_{1}\right) \neq\left(x_{2}, y_{2}\right)} \frac{\left.\mid \partial_{(x, y)} Y_{r}\left(x_{1}, y_{1}\right)\right)-\partial_{(x, y)} Y_{r}\left(x_{2}, y_{2}\right) \mid}{\left(\left|x_{1}-x_{2}\right|+\left|y_{1}-y_{2}\right|\right)^{\alpha}}
$$

remain bounded as $r \rightarrow 0$.

Proof: Since we have a symmetry between the variables $x$ and $y$ it is sufficient to consider only the derivative with respect to $x$. In the foregoing calculation we add and subtract convenient terms.

$$
\begin{aligned}
& \partial_{x} X_{r}(x, y)=\frac{1}{r} \partial_{x} \Phi\left(\frac{x}{r}, \frac{y}{r}\right) X(x, y)+\Phi\left(\frac{x}{r}, \frac{y}{r}\right) \partial_{x} X(x, y) \\
& \left|\frac{1}{r} \partial_{x} \Phi\left(\frac{x_{1}}{r}, \frac{y_{1}}{r}\right) X\left(x_{1}, y_{1}\right)+\Phi\left(\frac{x_{1}}{r}, \frac{y_{1}}{r}\right) \partial_{x} X\left(x_{1}, y_{1}\right)-\left[\frac{1}{r} \partial_{x} \Phi\left(\frac{x_{2}}{r}, \frac{y_{2}}{r}\right) X\left(x_{2}, y_{2}\right)+\Phi\left(\frac{x_{2}}{r}, \frac{y_{2}}{r}\right) \partial_{x} X\left(x_{2}, y_{2}\right)\right]\right| \\
& \leq\left|\frac{1}{r} \partial_{x} \Phi\left(\frac{x_{1}}{r}, \frac{y_{1}}{r}\right) X\left(x_{1}, y_{1}\right)-\frac{1}{r} \partial_{x} \Phi\left(\frac{x_{2}}{r}, \frac{y_{2}}{r}\right) X\left(x_{2}, y_{2}\right)\right|+\left|\Phi\left(\frac{x_{1}}{r}, \frac{y_{1}}{r}\right) \partial_{x} X\left(x_{1}, y_{1}\right)-\Phi\left(\frac{x_{2}}{r}, \frac{y_{2}}{r}\right) \partial_{x} X\left(x_{2}, y_{2}\right)\right| \leq \\
& \left|\frac{1}{r} \partial_{x} \Phi\left(\frac{x_{1}}{r}, \frac{y_{1}}{r}\right) X\left(x_{1}, y_{1}\right)-\frac{1}{r} \partial_{x} \Phi\left(\frac{x_{2}}{r}, \frac{y_{2}}{r}\right) X\left(x_{1}, y_{1}\right)+\frac{1}{r} \partial_{x} \Phi\left(\frac{x_{2}}{r}, \frac{y_{2}}{r}\right) X\left(x_{1}, y_{1}\right)-\frac{1}{r} \partial_{x} \Phi\left(\frac{x_{2}}{r}, \frac{y_{2}}{r}\right) X\left(x_{2}, y_{2}\right)\right| \\
& +\left|\Phi\left(\frac{x_{1}}{r}, \frac{y_{1}}{r}\right) \partial_{x} X\left(x_{1}, y_{1}\right)-\Phi\left(\frac{x_{2}}{r}, \frac{y_{2}}{r}\right) \partial_{x} X\left(x_{1}, y_{1}\right)+\Phi\left(\frac{x_{2}}{r}, \frac{y_{2}}{r}\right) \partial_{x} X\left(x_{1}, y_{1}\right)-\Phi\left(\frac{x_{2}}{r}, \frac{y_{2}}{r}\right) \partial_{x} X\left(x_{2}, y_{2}\right)\right| \leq \\
& \frac{1}{r}\left|\partial_{x} \Phi\left(\frac{x_{1}}{r}, \frac{y_{1}}{r}\right)-\partial_{x} \Phi\left(\frac{x_{2}}{r}, \frac{y_{2}}{r}\right)\right|\left|X\left(x_{1}, y_{1}\right)\right|+\frac{1}{r}\left|\partial_{x} \Phi\left(\frac{x_{2}}{r}, \frac{y_{2}}{r}\right)\right|\left|X\left(x_{1}, y_{1}\right)-X\left(x_{2}, y_{2}\right)\right|+ \\
& \quad+\left|\Phi\left(\frac{x_{1}}{r}, \frac{y_{1}}{r}\right)-\Phi\left(\frac{x_{2}}{r}, \frac{y_{2}}{r}\right)\right|\left|\partial_{x} X\left(x_{1}, y_{1}\right)\right|+\left|\Phi\left(\frac{x_{2}}{r}, \frac{y_{2}}{r}\right)\right|\left|\partial_{x} X\left(x_{1}, y_{1}\right)-\partial_{x} X\left(x_{2}, y_{2}\right)\right|
\end{aligned}
$$

To estimate

$$
\frac{\left|\partial_{x} X_{r}\left(x_{1}, y_{1}\right)-\partial_{x} X_{r}\left(x_{2}, y_{2}\right)\right|}{\left(\left|x_{1}-x_{2}\right|+\left|y_{1}-y_{2}\right|\right)^{\alpha}}
$$

we may suppose that $\left(x_{1}, y_{1}\right),\left(x_{1}, y_{1}\right)$ lie inside the ball or radius $r$. In fact, let us suppose that $\left(x_{1}, y_{1}\right)$ lies inside the ball or radius $r$ and $\left(x_{2}, y_{2}\right)$ lies outside. Let $\left(\bar{x}_{2}, \bar{y}_{2}\right)$ be a point in the sphere of radius $r$, that belongs to segment defined by $\left(x_{1}, y_{1}\right),\left(x_{2}, y_{2}\right)$. Then

$$
\begin{gathered}
\frac{\left|\partial_{x} X_{r}\left(x_{1}, y_{1}\right)-\partial_{x} X_{r}\left(x_{2}, y_{2}\right)\right|}{\left(\left|x_{1}-x_{2}\right|+\left|y_{1}-y_{2}\right|\right)^{\alpha}}=\frac{\left|\partial_{x} X_{r}\left(x_{1}, y_{1}\right)-\partial_{x} X_{r}\left(\bar{x}_{2}, \bar{y}_{2}\right)\right|}{\left(\left|x_{1}-\bar{x}_{2}\right|+\left|y_{1}-\bar{y}_{2}\right|\right)^{\alpha}} \frac{\left(\left|x_{1}-\bar{x}_{2}\right|+\left|y_{1}-\bar{y}_{2}\right|\right)^{\alpha}}{\left(\left|x_{1}-x_{2}\right|+\left|y_{1}-y_{2}\right|\right)^{\alpha}} \leq \\
\frac{\left|\partial_{x} X_{r}\left(x_{1}, y_{1}\right)-\partial_{x} X_{r}\left(\bar{x}_{2}, \bar{y}_{2}\right)\right|}{\left(\left|x_{1}-\bar{x}_{2}\right|+\left|y_{1}-\bar{y}_{2}\right|\right)^{\alpha}}
\end{gathered}
$$

Therefore,

$$
\frac{\left|\partial_{x} X_{r}\left(x_{1}, y_{1}\right)-\partial_{x} X_{r}\left(x_{2}, y_{2}\right)\right|}{\left(\left|x_{1}-x_{2}\right|+\left|y_{1}-y_{2}\right|\right)^{\alpha}} \leq
$$




$$
\begin{gathered}
\frac{1}{\left(\left|x_{1}-x_{2}\right|+\left|y_{1}-y_{2}\right|\right)^{\alpha}} \frac{1}{r}\left|\partial_{x} \Phi\left(\frac{x_{1}}{r}, \frac{y_{1}}{r}\right)-\partial_{x} \Phi\left(\frac{x_{2}}{r}, \frac{y_{2}}{r}\right)\right|\left|X\left(x_{1}, y_{1}\right)\right|+ \\
\frac{1}{\left(\left|x_{1}-x_{2}\right|+\left|y_{1}-y_{2}\right|\right)^{\alpha}} \frac{1}{r}\left|\partial_{x} \Phi\left(\frac{x_{2}}{r}, \frac{y_{2}}{r}\right)\right|\left|X\left(x_{1}, y_{1}\right)-X\left(x_{2}, y_{2}\right)\right|+ \\
+\frac{1}{\left(\left|x_{1}-x_{2}\right|+\left|y_{1}-y_{2}\right|\right)^{\alpha}}\left|\Phi\left(\frac{x_{1}}{r}, \frac{y_{1}}{r}\right)-\Phi\left(\frac{x_{2}}{r}, \frac{y_{2}}{r}\right)\right|\left|\partial_{x} X\left(x_{1}, y_{1}\right)\right| \\
+\frac{1}{\left(\left|x_{1}-x_{2}\right|+\left|y_{1}-y_{2}\right|\right)^{\alpha}}\left|\Phi\left(\frac{x_{2}}{r}, \frac{y_{2}}{r}\right)\right|\left|\partial_{x} X\left(x_{1}, y_{1}\right)-\partial_{x} X\left(x_{2}, y_{2}\right)\right|
\end{gathered}
$$

Now we are going to prove that each adding term of the above expression remains bounded.

We start with (2.2).

$$
\begin{gathered}
\frac{1}{\left(\left|x_{1}-x_{2}\right|+\left|y_{1}-y_{2}\right|\right)^{\alpha}} \frac{1}{r}\left|\partial_{x} \Phi\left(\frac{x_{1}}{r}, \frac{y_{1}}{r}\right)-\partial_{x} \Phi\left(\frac{x_{2}}{r}, \frac{y_{2}}{r}\right)\right|\left|X\left(x_{1}, y_{1}\right)\right| \leq \\
\frac{1}{r} \frac{\left|X\left(x_{1}, y_{1}\right)\right|}{\left(\left|x_{1}-x_{2}\right|+\left|y_{1}-y_{2}\right|\right)^{\alpha}}\left|\partial_{x} \Phi\left(\frac{x_{1}}{r}, \frac{y_{1}}{r}\right)-\partial_{x} \Phi\left(\frac{x_{2}}{r}, \frac{y_{2}}{r}\right)\right| \leq \\
\frac{\text { const. }}{r} \frac{\left|X\left(x_{1}, y_{1}\right)\right|}{\left(\left|x_{1}-x_{2}\right|+\left|y_{1}-y_{2}\right|\right)^{\alpha}} \frac{\left(\left|x_{1}-x_{2}\right|+\left|y_{1}-y_{2}\right|\right)}{r} \leq \frac{\text { const. }}{r^{2}}\left|X\left(x_{1}, y_{1}\right)\right|\left[\left|x_{1}-x_{2}\right|+\left|y_{1}-y_{2}\right|\right]^{(1-\alpha)} \leq \\
\leq \frac{\text { const. }}{r^{2}}\left|X\left(x_{1}, y_{1}\right)\right| r^{(1-\alpha)} \leq \frac{\text { const. }}{r^{(1+\alpha)}}\left|X\left(x_{1}, y_{1}\right)\right| \leq \frac{\text { const. }}{r^{(1+\alpha)} \mid} \int_{0}^{1} \frac{d}{d t} X\left(t x_{1}, t y_{1}\right) d t \mid \leq \\
\left.\frac{\text { const. }}{r^{(1+\alpha)}} \int_{0}^{1}\left[\left|\partial_{x} X\left(t x_{1}, t y_{1}\right)\right|\left|x_{1}\right|+\mid \partial_{y} X\left(t x_{1}, \text { ty } y_{1}\right)|| y_{1} \mid\right] d t \leq \frac{\text { const. }}{r^{\alpha}} \int_{0}^{1}\left[\left|t x_{1}\right|+\left|t y_{1}\right|\right]^{\alpha}\right] d t \leq \\
\left.\frac{\text { const. }}{r^{\alpha}} \int_{0}^{1}\left[\left|t x_{1}\right|^{\alpha}+\left|t y_{1}\right|^{\alpha}\right]\right] d t \leq \text { const. } \int_{0}^{1} t^{\alpha} d t \leq \text { const. }
\end{gathered}
$$

Now we are going to estimate (2.3).

$$
\begin{gathered}
\quad \frac{1}{\left(\left|x_{1}-x_{2}\right|+\left|y_{1}-y_{2}\right|\right)^{\alpha}} \frac{1}{r}\left|\partial_{x} \Phi\left(\frac{x_{2}}{r}, \frac{y_{2}}{r}\right)\right|\left|X\left(x_{1}, y_{1}\right)-X\left(x_{2}, y_{2}\right)\right| \leq \\
\frac{1}{r}\left|\partial_{x} \Phi\left(\frac{x_{2}}{r}, \frac{y_{2}}{r}\right)\right| \frac{1}{\left(\left|x_{1}-x_{2}\right|+\left|y_{1}-y_{2}\right|\right)^{\alpha}}\left|X\left(x_{1}, y_{1}\right)-X\left(x_{2}, y_{2}\right)\right| \leq \\
\quad \frac{\text { const. }}{r} \frac{1}{\left(\left|x_{1}-x_{2}\right|+\left|y_{1}-y_{2}\right|\right)^{\alpha}}\left|X\left(x_{1}, y_{1}\right)-X\left(x_{2}, y_{2}\right)\right| \leq \\
\text { const. } \left.\frac{1}{r} \frac{1}{\left(\left|x_{1}-x_{2}\right|+\left|y_{1}-y_{2}\right|\right)^{\alpha}} \| \int_{0}^{1} \frac{d}{d t} X\left(t x_{1}+(1-t) x_{2}\right), t y_{1}+(1-t) y_{2}\right) d t \mid \leq
\end{gathered}
$$




$$
\begin{aligned}
& \frac{\text { const. }}{r} \frac{\left.\left.\mid \int_{0}^{1} \partial_{x} X\left(t x_{1}+(1-t) x_{2}\right), t y_{1}+(1-t) y_{2}\right) d t|| x_{1}-x_{2}|+| \int_{0}^{1} \partial_{y} X\left(t x_{1}+(1-t) x_{2}\right), t y_{1}+(1-t) y_{2}\right) d t|| y_{1}-y_{2} \mid}{\left(\left|x_{1}-x_{2}\right|+\left|y_{1}-y_{2}\right|\right)^{\alpha}} \\
& \quad \leq \frac{\text { const. }}{r} \frac{\left.\int_{0}^{1}\left[\left|t x_{1}+(1-t) x_{2}\right|+\mid t y_{1}+(1-t) y_{2}\right) \mid\right]^{\alpha} d t\left(\left|x_{1}-x_{2}\right|+\left|y_{1}-y_{2}\right|\right)}{\left(\left|x_{1}-x_{2}\right|+\left|y_{1}-y_{2}\right|\right)^{\alpha}} \leq \frac{\text { const. }}{r^{\alpha}} r^{\alpha} \leq \text { const. }
\end{aligned}
$$

Next we will estimate (2.4).

$$
\begin{gathered}
\frac{1}{\left(\left|x_{1}-x_{2}\right|+\left|y_{1}-y_{2}\right|\right)^{\alpha}}\left|\Phi\left(\frac{x_{1}}{r}, \frac{y_{1}}{r}\right)-\Phi\left(\frac{x_{2}}{r}, \frac{y_{2}}{r}\right)\right|\left|\partial_{x} X\left(x_{1}, y_{1}\right)\right|= \\
\frac{\left|\partial_{x} X\left(x_{1}, y_{1}\right)\right|}{\left(\left|x_{1}-x_{2}\right|+\left|y_{1}-y_{2}\right|\right)^{\alpha}}\left|\int_{0}^{1} \frac{d}{d t} \Phi\left(\frac{t x_{1}+(1-t) x_{2}}{r}, \frac{t y_{1}+(1-t) y_{2}}{r}\right) d t\right|= \\
\left|\partial_{x} X\left(x_{1}, y_{1}\right)\right| \frac{\left|\int_{0}^{1}\left[\partial_{x} \Phi\left(\frac{t x_{1}+(1-t) x_{2}}{r}, \frac{t y_{1}+(1-t) y_{2}}{r}\right) \frac{\left(x_{1}-x_{2}\right)}{r}+\partial_{y} \Phi\left(\frac{t x_{1}+(1-t) x_{2}}{r}, \frac{t y_{1}+(1-t) y_{2}}{r}\right) \frac{\left(y_{1}-y_{2}\right)}{r}\right] d t\right|}{\left(\left|x_{1}-x_{2}\right|+\left|y_{1}-y_{2}\right|\right)^{\alpha}} \\
\leq \frac{\text { const. }\left|\partial_{x} X\left(x_{1}, y_{1}\right)\right|}{\left(\left|x_{1}-x_{2}\right|+\left|y_{1}-y_{2}\right|\right)^{\alpha}}\left(\frac{\left|x_{1}-x_{2}\right|+\left|y_{1}-y_{2}\right|}{r}\right) \leq \frac{\text { const. }\left|\partial_{x} X\left(x_{1}, y_{1}\right)\right|}{r}\left(\left|x_{1}-x_{2}\right|+\left|y_{1}-y_{2}\right|\right)^{(1-\alpha)} \\
\leq \frac{\text { const. }\left|\partial_{x} X\left(x_{1}, y_{1}\right)\right|}{r^{\alpha}} \leq \frac{\text { const. }\left|\left(\left|x_{1}\right|+\left|x_{2}\right|\right)\right|^{\alpha}}{r^{\alpha}} \leq \text { const. }
\end{gathered}
$$

It is left to estimate $(2.5)$.

$$
\begin{gathered}
\frac{1}{\left(\left|x_{1}-x_{2}\right|+\left|y_{1}-y_{2}\right|\right)^{\alpha}}\left|\Phi\left(\frac{x_{2}}{r}, \frac{y_{2}}{r}\right)\right|\left|\partial_{x} X\left(x_{1}, y_{1}\right)-\partial_{x} X\left(x_{2}, y_{2}\right)\right| \leq \\
\text { const. } \frac{\left|\partial_{x} X\left(x_{1}, y_{1}\right)-\partial_{x} X\left(x_{2}, y_{2}\right)\right|}{\left(\left|x_{1}-x_{2}\right|+\left|y_{1}-y_{2}\right|\right)^{\alpha}} \leq \text { const. }
\end{gathered}
$$

To solve the functional equations that will appear in the next section, we will have to look for the solutions in some suitable space, that we introduce next.

Let $\mathbb{X}_{1}, \mathbb{X}_{2}, \mathbb{X}_{3}$ be Banach spaces. Consider the normed space:

$$
\mathcal{E}_{0}^{1, \alpha}=:\left\{f \in \mathcal{C}^{1}\left(\mathbb{X}_{1} \times \mathbb{X}_{2}, \mathbb{X}_{3}\right): f(\xi, 0) \equiv 0, f(0, \eta) \equiv 0, \text { and }\|f\|_{\alpha}<\infty\right\}
$$

where,

$$
\|f\|_{\alpha}=: \max \left\{\sup _{\eta \neq 0} \frac{\left|\partial_{\xi} f(\xi, \eta)\right|}{|\eta|^{\alpha}}, \sup _{\xi \neq 0} \frac{\left|\partial_{\eta} f(\xi, \eta)\right|}{|\xi|^{\alpha}}\right\}
$$

Lemma 2. $\mathcal{E}_{0}^{1, \alpha}$ with the norm $\|\cdot\|_{\alpha}$ is a Banach space. 
Proof: Let $\left(f_{n}\right)_{n \in \mathbb{N}}$ be a Cauchy sequence in $\mathcal{E}_{0}^{1, \alpha}$. Given $\varepsilon>0$, there exists $n_{0}$, such that, $m, n \geq n_{0} \Rightarrow\left\|f_{n}-f_{m}\right\|_{\alpha}<\varepsilon$, which implies:

$$
\left|\partial_{\xi} f_{n}(\xi, \eta)-\partial_{\xi} f_{m}(\xi, \eta)\right| \leq \varepsilon|\eta|^{\alpha}, \quad\left|\partial_{\eta} f_{n}(\xi, \eta)-\partial_{\eta} f_{m}(\xi, \eta)\right| \leq \varepsilon|\xi|^{\alpha}
$$

for every $(\xi, \eta) \in \mathbb{X}_{1} \times \mathbb{X}_{2}$.

Therefore, there exist $F, G: \mathbb{X}_{1} \times \mathbb{X}_{2} \rightarrow \mathbb{X}_{3}$, such that $\partial_{\xi} f_{n} \rightarrow F, \partial_{\eta} f_{n} \rightarrow G$, uniformly on bounded sets of $\mathbb{X}_{1} \times \mathbb{X}_{2}$.

Our purpose is to show that there exists $f: \mathbb{X}_{1} \times \mathbb{X}_{2} \rightarrow \mathbb{X}_{3}$, such that $\left\|f_{n}-f\right\|_{\alpha} \rightarrow 0$, as $n \rightarrow \infty$.

For $t \in[0,1]$ we have that $\frac{d f_{n}(t \xi, \eta)}{d t}=\partial_{\xi} f_{n}(t \xi, \eta) \xi$ and so,

$$
f_{n}(\xi, \eta)=f_{n}(\xi, \eta)-f_{n}(\xi, 0)=\int_{0}^{1} \frac{d f_{n}(t \xi, \eta)}{d t} d t=\int_{0}^{1} \partial_{\xi} f_{n}(t \xi, \eta) \xi d t
$$

Therefore

$$
f_{n}(\xi, \eta)=\int_{0}^{1} \partial_{\xi} f_{n}(t \xi, \eta) \xi d t \rightarrow f(\xi, \eta)=: \int_{0}^{1} F(t \xi, \eta) \xi d t
$$

as $n \rightarrow \infty$, uniformly on bounded sets of $\mathbb{X}_{1} \times \mathbb{X}_{2}$.

A similar idea gives:

$$
f_{n}(\xi, \eta)=\int_{0}^{1} \partial_{\eta} f_{n}(t \xi, \eta) \eta d t \rightarrow f(\xi, \eta)=\int_{0}^{1} G(\xi, t \eta) \eta d t
$$

as $n \rightarrow \infty$, uniformly on bounded sets of $\mathbb{X}_{1} \times \mathbb{X}_{2}$.

One can easily see that $f(\xi, 0)=f(0, \eta) \equiv 0$.

Next we are going to prove that $f$ is $C^{1}$.

For $\left(\xi_{0}, \eta_{0}\right) \in \mathbb{X}_{1} \times \mathbb{X}_{2}$, we claim that $\frac{1}{|h|}\left[f_{n}\left(\xi_{0}+h, \eta_{0}\right)-f_{n}\left(\xi_{0}, \eta_{0}\right)\right]$ is a Cauchy sequence for $h \neq 0$.

In fact,

$$
\begin{gathered}
{\left[f_{n}\left(\xi_{0}+h, \eta_{0}\right)-f_{n}\left(\xi_{0}, \eta_{0}\right)-f_{m}\left(\xi_{0}+h, \eta_{0}\right)+f_{m}\left(\xi_{0}, \eta_{0}\right)\right]=} \\
\int_{0}^{1} \frac{d}{d \theta}\left[f_{n}\left(\xi_{0}+\theta h, \eta_{0}\right)-f_{m}\left(\xi_{0}+\theta h, \eta_{0}\right)\right] d \theta=\int_{0}^{1}\left[\partial_{\xi} f_{n}\left(\xi_{0}+\theta h, \eta_{0}\right)-\partial_{\xi} f_{m}\left(\xi_{0}+\theta h, \eta_{0}\right)\right] h d \theta
\end{gathered}
$$

From (2.7) it follows that, for $m, n \geq n_{0}$ :

$$
\frac{1}{|h|}\left|\left[f_{n}\left(\xi_{0}+h, \eta_{0}\right)-f_{n}\left(\xi_{0}, \eta_{0}\right)-f_{m}\left(\xi_{0}+h, \eta_{0}\right)+f_{m}\left(\xi_{0}, \eta_{0}\right)\right]\right| \leq
$$




$$
\int_{0}^{1}\left|\partial_{\xi} f_{n}\left(\xi_{0}+\theta h, \eta_{0}\right)-\partial_{\xi} f_{m}\left(\xi_{0}+\theta h, \eta_{0}\right)\right| d \theta \leq \varepsilon\left|\eta_{0}\right|^{\alpha} .
$$

If we let $m \rightarrow \infty$ in the above inequality, we obtain, for $n \geq n_{0}$ :

$$
\frac{1}{|h|}\left|\left[f_{n}\left(\xi_{0}+h, \eta_{0}\right)-f_{n}\left(\xi_{0}, \eta_{0}\right)-f\left(\xi_{0}+h, \eta_{0}\right)+f\left(\xi_{0}, \eta_{0}\right)\right]\right| \leq \varepsilon\left|\eta_{0}\right|^{\alpha} .
$$

Now are ready to prove that $f \in C^{1}$.

$$
\begin{gathered}
\frac{1}{|h|}\left|f\left(\xi_{0}+h, \eta_{0}\right)-f\left(\xi_{0}, \eta_{0}\right)-F\left(\xi_{0}, \eta_{0}\right) h\right| \leq \\
\frac{1}{|h|}\left|f\left(\xi_{0}+h, \eta_{0}\right)-f\left(\xi_{0}, \eta_{0}\right)-\left(f_{n_{0}}\left(\xi_{0}+h, \eta_{0}\right)-f_{n_{0}}\left(\xi_{0}, \eta_{0}\right)\right)\right|+ \\
\left.\frac{1}{|h|} \mid\left[f_{n_{0}}\left(\xi_{0}+h, \eta_{0}\right)-f_{n_{0}}\left(\xi_{0}, \eta_{0}\right)\right)-\partial_{\xi} f_{n_{0}}\left(\xi_{0}, \eta_{0}\right) h\right]+\left[\partial_{\xi} f_{n_{0}}\left(\xi_{0}, \eta_{0}\right) h-F\left(\xi_{0}, \eta_{0}\right) h\right] \mid \leq \\
\left.\left.\left.\varepsilon\left|\eta_{0}\right|^{\alpha}+\frac{1}{|h|} \mid f_{n_{0}}\left(\xi_{0}+h, \eta_{0}\right)-f_{n_{0}}\left(\xi_{0}, \eta_{0}\right)\right)-\partial_{\xi} f_{n_{0}}\left(\xi_{0}, \eta_{0}\right) h|+| \partial_{\xi} f_{n_{0}}\left(\xi_{0}, \eta_{0}\right)\right)-F\left(\xi_{0}, \eta_{0}\right)\right] \mid
\end{gathered}
$$

Choosing a larger $n_{0}$ if necessary, we can suppose that $\left.\mid \partial_{\xi} f_{n_{0}}\left(\xi_{0}, \eta_{0}\right)-F\left(\xi_{0}, \eta_{0}\right)\right] \mid<\varepsilon$.

Our assumptions imply that there exists $\delta>0$, such that:

$$
\left.|h|<\delta \Rightarrow \frac{1}{|h|} \mid f_{n_{0}}\left(\xi_{0}+h, \eta_{0}\right)-f_{n_{0}}\left(\xi_{0}, \eta_{0}\right)\right)-\partial_{\xi} f_{n_{0}}\left(\xi_{0}, \eta_{0}\right) h \mid<\varepsilon .
$$

Therefore,

$$
|h|<\delta \Rightarrow \frac{1}{|h|}\left|f\left(\xi_{0}+h, \eta_{0}\right)-f\left(\xi_{0}, \eta_{0}\right)-F\left(\xi_{0}, \eta_{0}\right) h\right|<\varepsilon
$$

This proves that $\partial_{\xi} f\left(\xi_{0}, \eta_{0}\right)=F\left(\xi_{0}, \eta_{0}\right)$.

In a similar way we can prove that $\partial_{\eta} f\left(\xi_{0}, \eta_{0}\right)=G\left(\xi_{0}, \eta_{0}\right)$. Since $f$ has continuous partial derivatives, we conclude that $f \in C^{1}\left(\mathbb{X}_{1} \times \mathbb{X}_{2}, \mathbb{X}_{3}\right)$.

If we let $n=n_{0}$ and let $m \rightarrow \infty$ in (2.7), we obtain for every $(\xi, \eta) \in \mathbb{X}_{1} \times \mathbb{X}_{2}$ :

$$
\begin{gathered}
\left|\partial_{\xi} f_{n_{0}}(\xi, \eta)-F(\xi, \eta)\right|=\left|\partial_{\xi} f_{n_{0}}(\xi, \eta)-\partial_{\xi} f(\xi, \eta)\right| \leq \varepsilon|\eta|^{\alpha} \\
\left|\partial_{\eta} f_{n_{0}}(\xi, \eta)-G(\xi, \eta)\right|=\left|\partial_{\eta} f_{n_{0}}(\xi, \eta)-\partial_{\eta} f(\xi, \eta)\right| \leq \varepsilon|\xi|^{\alpha},
\end{gathered}
$$

which implies that $f-f_{n_{0}} \in \mathcal{E}_{0}^{1, \alpha}$ and so $f \in \mathcal{E}_{0}^{1, \alpha}$.

If we let $m \rightarrow \infty$ in (2.7), we obtain for every $(\xi, \eta) \in \mathbb{X}_{1} \times \mathbb{X}_{2}, n \geq n_{0}$ :

$$
\begin{gathered}
\left|\partial_{\xi} f_{n}(\xi, \eta)-F(\xi, \eta)\right|=\left|\partial_{\xi} f_{n}(\xi, \eta)-\partial_{\xi} f(\xi, \eta)\right| \leq \varepsilon|\eta|^{\alpha} \\
\left|\partial_{\eta} f_{n}(\xi, \eta)-G(\xi, \eta)\right|=\left|\partial_{\eta} f_{n}(\xi, \eta)-\partial_{\eta} f(\xi, \eta)\right| \leq \varepsilon|\xi|^{\alpha}
\end{gathered}
$$


which imply that $f_{n} \rightarrow f$ in $\mathcal{E}_{0}^{1, \alpha}$.

\section{Functional Equations.}

The following lemmas will be used in the proof of our main theorem.

Lemma 3. Suppose $A, L \in \mathcal{L}(X), F(x)=: L x+f(x)$ a local mapping, such that, $f(0)=0, \partial_{\xi} f(0)=0$ and $f \in \mathcal{C}^{1, \alpha}$ locally, where $0<\alpha \leq 1$. If $|A||L|^{(1+\alpha)}<1$ then the functional equation:

$$
\phi(\xi)=A \phi(L \xi+f(\xi))-A f(\xi)
$$

has a solution $\phi \in \mathcal{C}^{1, \alpha}$ locally, such that $\phi(0)=0, \partial_{\xi} \phi(0)=0$.

Proof: To extend our local nonlinearity to the whole space we let $f_{r}(\xi)=: \Phi(\xi / r) f(\xi)$, where $\Phi$ satisfies (2.1) and $r$ is sufficiently small.

Now consider the equation:

$$
\phi(\xi)=A \phi\left(L \xi+f_{r}(\xi)\right)-A f_{r}(\xi)
$$

We will prove that if $r$ is sufficiently small, then there exists a unique solution $\phi$ of (3.2), such that $\phi(0)=0, \partial_{\xi} \phi(0)=0, \phi \in \mathcal{C}^{1, \alpha}(X, X)$.

We consider the following Banach space:

$$
\mathcal{E}=\mathcal{E}_{\alpha}=:\left\{\phi \in \mathcal{C}^{1, \alpha}(X, X): \phi(0)=0, \partial_{\xi} \phi(0)=0\right\}
$$

with the norm: $\|\phi\|=: \sup _{\xi_{1} \neq \xi_{2}} \frac{\left|\partial_{\xi} \phi\left(\xi_{1}\right)-\partial_{\xi} \phi\left(\xi_{2}\right)\right|}{\left|\xi_{1}-\xi_{2}\right|^{\alpha}}$.

To solve (3.2) we will use the Contraction Principle. We will prove that the linear operator $T: \mathcal{E} \rightarrow \mathcal{E},(T \phi)(\xi)=: A \phi\left(L \xi+f_{r}(\xi)\right)$ is a contraction on $\mathcal{E}$.

Since, $\partial_{\xi}(T \phi)(\xi)=A \partial_{\xi} \phi\left(L \xi+f_{r}(\xi)\right)\left(L+\partial_{\xi} f_{r}(\xi)\right)$, we have:

$$
\begin{gathered}
\frac{\left|\partial_{\xi}(T \phi)\left(\xi_{1}\right)-\partial_{\xi}(T \phi)\left(\xi_{2}\right)\right|}{\left|\xi_{1}-\xi_{2}\right|^{\alpha}}= \\
\frac{\left|A \partial_{\xi} \phi\left(L \xi_{1}+f_{r}\left(\xi_{1}\right)\right)\left(L+\partial_{\xi} f_{r}\left(\xi_{1}\right)\right)-A \partial_{\xi} \phi\left(L \xi_{2}+f_{r}\left(\xi_{2}\right)\right)\left(L+\partial_{\xi} f_{r}\left(\xi_{2}\right)\right)\right|}{\left|\xi_{1}-\xi_{2}\right|^{\alpha}}= \\
\frac{\left|A\left[\partial_{\xi} \phi\left(L \xi_{1}+f_{r}\left(\xi_{1}\right)\right)-\partial_{\xi} \phi\left(L \xi_{2}+f_{r}\left(\xi_{2}\right)\right)\right]\left(L+\partial_{\xi} f_{r}\left(\xi_{1}\right)\right)+A \partial_{\xi} \phi\left(L \xi_{2}+f_{r}\left(\xi_{2}\right)\right)\left[\left(L+\partial_{\xi} f_{r}\left(\xi_{1}\right)\right)-\left(L+\partial_{\xi} f_{r}\left(\xi_{2}\right)\right)\right]\right|}{\left|\xi_{1}-\xi_{2}\right|^{\alpha}}
\end{gathered}
$$




$$
\begin{gathered}
\leq \frac{|A|\left|\partial_{\xi} \phi\left(L \xi_{1}+f_{r}\left(\xi_{1}\right)\right)-\partial_{\xi} \phi\left(L \xi_{2}+f_{r}\left(\xi_{2}\right)\right)\right|\left|L+\partial_{\xi} f_{r}\left(\xi_{1}\right)\right|}{\left|\xi_{1}-\xi_{2}\right|^{\alpha}}+ \\
\frac{|A|\left|\partial_{\xi} \phi\left(L \xi_{2}+f_{r}\left(\xi_{2}\right)\right)\right|\left|\left(L+\partial_{\xi} f_{r}\left(\xi_{1}\right)\right)-\left(L+\partial_{\xi} f_{r}\left(\xi_{2}\right)\right)\right|}{\left|\xi_{1}-\xi_{2}\right|^{\alpha}} \leq \\
\frac{|A|\|\phi\|\left|\left(L \xi_{1}+f_{r}\left(\xi_{1}\right)\right)-\left(L \xi_{2}+f_{r}\left(\xi_{2}\right)\right)\right|^{\alpha}\left|L+\partial_{\xi} f_{r}\left(\xi_{1}\right)\right|}{\left|\xi_{1}-\xi_{2}\right|^{\alpha}}+ \\
|A|\|\phi\||| L \xi_{2}+\left.f_{r}\left(\xi_{2}\right)\right|^{\alpha} \frac{\left|\left(\partial_{\xi} f_{r}\left(\xi_{1}\right)-\partial_{\xi} f_{r}\left(\xi_{2}\right)\right)\right|}{\left|\xi_{1}-\xi_{2}\right|^{\alpha}} \leq \\
|A|\|\phi\|\left|L+\partial_{\xi} f_{r}\left(\xi_{1}\right)\right| \frac{\left|\left(L\left(\xi_{1}-\xi_{2}\right)+\left(f_{r}\left(\xi_{1}\right)\right)-f_{r}\left(\xi_{2}\right)\right)\right|^{\alpha}}{\left|\xi_{1}-\xi_{2}\right|^{\alpha}}+ \\
|A|\|\phi\||| L \xi_{2}+\left.f_{r}\left(\xi_{2}\right)\right|^{\alpha} \frac{\left|\left(\partial_{\xi} f_{r}\left(\xi_{1}\right)-\partial_{\xi} f_{r}\left(\xi_{2}\right)\right)\right|}{\left|\xi_{1}-\xi_{2}\right|^{\alpha}}
\end{gathered}
$$

Next we are going to estimate (3.3).

$$
\begin{gathered}
|A| \| \phi||\left|L+\partial_{\xi} f_{r}\left(\xi_{1}\right)\right| \frac{\left|\left(L\left(\xi_{1}-\xi_{2}\right)+\left(f_{r}\left(\xi_{1}\right)\right)-f_{r}\left(\xi_{2}\right)\right)\right|^{\alpha}}{\left|\xi_{1}-\xi_{2}\right|^{\alpha}} \leq \\
|A|\|\phi\|\left[|L|+\left|\partial_{\xi} f_{r}\left(\xi_{1}\right)\right|\right]|| L\left|+\frac{\left.\mid f_{r}\left(\xi_{1}\right)\right)-f_{r}\left(\xi_{2}\right) \mid}{\left|\xi_{1}-\xi_{2}\right|}\right|^{\alpha} \leq \\
|A|\|\phi\|\left[|L|+\left|\partial_{\xi} f_{r}\left(\xi_{1}\right)\right|\right]|| L\left|+\frac{\left|\int_{0}^{1} \frac{d}{d t} f_{r}\left(t \xi_{1}+(1-t) \xi_{2}\right) d t\right|}{\left|\xi_{1}-\xi_{2}\right|}\right|^{\alpha} \mid \\
\leq|A|\|\phi\|\left[|L|+O\left(r^{\alpha}\right)\right]|| L\left|+\frac{\left|\int_{0}^{1} \partial_{\xi} f_{r}\left(t \xi_{1}+(1-t) \xi_{2}\right) d t\left(\xi_{1}-\xi_{2}\right)\right|}{\left|\xi_{1}-\xi_{2}\right|}\right|^{\alpha} \leq \\
\left.|A|\|\phi\|\left[|L|+O\left(r^{\alpha}\right)\right]|| L\right|^{\alpha}+\left(O\left(r^{\alpha}\right)\right)^{\alpha} \mid
\end{gathered}
$$

Let us estimate (3.4). If $\left|\xi_{2}\right| \leq 2 r$, we have:

$$
|A|\|\phi\|\left|L \xi_{2}+f_{r}\left(\xi_{2}\right)\right|^{\alpha} \frac{\left|\left(\partial_{\xi} f_{r}\left(\xi_{1}\right)-\partial_{\xi} f_{r}\left(\xi_{2}\right)\right)\right|}{\left|\xi_{1}-\xi_{2}\right|^{\alpha}} \leq\|\phi\| O\left(r^{\alpha}\right) .
$$

If $\left|\xi_{1}\right| \geq r,\left|\xi_{2}\right|>2 r$, we have:

$$
|A| \| \phi||\left|L \xi_{2}+f_{r}\left(\xi_{2}\right)\right|^{\alpha} \frac{\left|\left(\partial_{\xi} f_{r}\left(\xi_{1}\right)-\partial_{\xi} f_{r}\left(\xi_{2}\right)\right)\right|}{\left|\xi_{1}-\xi_{2}\right|^{\alpha}}=0 .
$$

If $\left|\xi_{1}\right|<r,\left|\xi_{2}\right|>2 r$, we have:

$$
|A|\|\phi\|\left|L \xi_{2}+f_{r}\left(\xi_{2}\right)\right|^{\alpha} \frac{\left|\left(\partial_{\xi} f_{r}\left(\xi_{1}\right)-\partial_{\xi} f_{r}\left(\xi_{2}\right)\right)\right|}{\left|\xi_{1}-\xi_{2}\right|^{\alpha}} \leq|A|\|\phi\|\left(\frac{\left|L \xi_{2}\right|}{\left|\xi_{2}\right|}\right)^{\alpha} \frac{\mid\left(\partial_{\xi} f_{r}\left(\xi_{1}\right) \mid\right.}{\left|\xi_{1}-\xi_{2}\right|^{\alpha}}\left|\xi_{2}\right|^{\alpha} \leq
$$




$$
|A|\left\|\phi \left|\left\|\left.L\right|^{\alpha} \frac{\mid\left(\partial_{\xi} f_{r}\left(\xi_{1}\right) \mid\right.}{\left|\xi_{1}\right|^{\alpha}}\left|\xi_{1}\right|^{\alpha}\left(\frac{\left|\xi_{2}\right|}{\left|\xi_{1}-\xi_{2}\right|}\right)^{\alpha} \leq\right\| \phi \| O\left(r^{\alpha}\right) .\right.\right.
$$

In the last inequality we used that $\frac{\left|\xi_{2}\right|}{\left|\xi_{1}-\xi_{2}\right|} \leq 2$.

In fact, since $\left|\xi_{1}\right|<r,\left|\xi_{2}\right|>2 r$, we have, $\frac{\left|\xi_{1}\right|}{\left|\xi_{2}\right|} \leq \frac{r}{2 r}=\frac{1}{2}$

Therefore,

$$
\frac{\left|\xi_{2}-\xi_{1}\right|}{\left|\xi_{2}\right|} \geq \frac{\left|\xi_{2}\right|}{\left|\xi_{2}\right|}-\frac{\left|\xi_{1}\right|}{\left|\xi_{2}\right|} \geq 1-\frac{1}{2}=\frac{1}{2}
$$

But this implies that $\frac{\left|\xi_{2}\right|}{\left|\xi_{1}-\xi_{2}\right|} \leq 2$.

The above calculation shows that: $\|T \phi\| \leq\left[|A \| L|^{1+\alpha}+O\left(r^{\alpha}\right)\right]\|\phi\|$.

Therefore, if we take $r$ sufficiently close to 0 , the hypotheses of our lemma imply that the operator $T$ will be a contraction.

The next theorem can be proved using Lemma 3. In some respects it can be seen as an extension of a result of X. Mora and J. Solà-Morales[4].

Theorem 4. Suppose $L \in \mathcal{L}(X)$ and that $F(x)=: L x+f(x)$ is a local mapping, such that, $f(0)=0, \partial_{\xi} f(0)=0$ and $f \in \mathcal{C}^{1, \alpha}, 0<\alpha \leq 1$, locally. Suppose that $|\sigma(L)| \subset(a, b)$, where $0<a<b<1$. If $b^{1+\alpha}<a$ then there exists a function $R=: I+\phi$, where $\phi \in \mathcal{C}^{1, \alpha}$, locally, $\phi(0)=0, \phi^{\prime}(0)=0$, such that $R F R^{-1}=L$.

The following lemma on regularity of invariants manifolds follows from the work of D. Henry[3].

Lemma 4. Let $X$ be a Banach space and $L \in \mathcal{L}(X)$. Suppose $|\sigma(L)| \subset(a, b) \cup(c, d)$, where $0<a<b<1<c<d$. Let $S$ and $U$, respectively, be the stable and unstable subspaces of L. Consider the local mapping $F(x)=: L x+f(x)$, where $f(0)=0, f^{\prime}(0)=0$ and $f \in \mathcal{C}^{1, \alpha}$, locally, where $0<\alpha \leq 1$.

Then the local stable and unstable manifolds for $F$ are of the form:

$$
M_{s}=:\left\{x=(\xi, \eta): \eta=\gamma_{s}(\xi)\right\}, \quad M_{u}=:\left\{x=(\xi, \eta): \xi=\gamma_{u}(\eta)\right\}
$$

with $\gamma_{s}$ and $\gamma_{u}$ also locally in the class $\mathcal{C}^{1, \alpha}$ and $\gamma_{s}(0)=0, \gamma_{s}^{\prime}(0)=0, \gamma_{u}(0)=0, \gamma_{u}^{\prime}(0)=0$. 
Let $X_{1}, X_{2}$ be Banach spaces and

$$
\mathcal{E}=\mathcal{E}_{0}^{1, \alpha}=:\left\{f \in \mathcal{C}^{1}\left(X_{1} \times X_{2}, X_{2}\right): f(\xi, 0) \equiv 0, f(0, \eta) \equiv 0, \text { and }\|f\|_{\alpha}<\infty\right\}
$$

where $\|f\|_{\alpha}$ was defined in (2.6).

Lemma 5. Let $A, B \in \mathcal{L}\left(X_{1}\right)$ and $C \in \mathcal{L}\left(X_{2}\right)$. Let $f^{1}: X_{1} \times X_{2} \rightarrow X_{1}, f^{2}: X_{1} \times X_{2} \rightarrow X_{2}$ be $C^{1}$ local functions such that $f^{1}(0, \eta)=f^{1}(\xi, 0)=0, f^{2}(0, \eta)=f^{2}(\xi, 0)=0$ and $f=\left(f^{1}, f^{2}\right)$. We assume that the space $X_{1} \times X_{2}$ satisfies the condition (1.1), for a certain function $\Phi$ and that $f_{r}(\xi, \eta)=: \Phi(\xi / r, \eta / r) f(\xi, \eta)$, for $r$ sufficiently small. Let $f_{r}(\xi, \eta)=\left(f_{r}^{1}(\xi, \eta), f_{r}^{2}(\xi, \eta)\right)$.

If $|A||B||C|<1, \alpha$ is sufficiently close to 1 and $\psi \in \mathcal{E}$, then the functional equation:

$$
\phi(\xi, \eta)=A \phi\left(B \xi+f_{r}^{1}(\xi, \eta), C \eta+f_{r}^{2}(\xi, \eta)\right)+\psi(\xi, \eta)
$$

has a solution $\phi \in \mathcal{E}$

Proof: Consider the following linear operator defined in $\mathcal{E}$,

$$
(T \phi)(\xi, \eta)=: A \phi\left(B \xi+f_{r}^{1}(\xi, \eta), C \eta+f_{r}^{2}(\xi, \eta)\right)
$$

Next we are going to prove that $\mathcal{E}$ is invariant under $T$ and that $T$ is a contraction if $r$ is sufficiently small and $\alpha$ is sufficiently close to 1 .

$$
\begin{gathered}
\frac{\left|\partial_{\xi}(T \phi)(\xi, \eta)\right|}{|\eta|^{\alpha}}=\frac{1}{|\eta|^{\alpha}}\left|\partial_{\xi}\left[A \phi\left(B \xi+f_{r}^{1}(\xi, \eta), C \eta+f_{r}^{2}(\xi, \eta)\right)\right]\right| \leq \\
\frac{1}{|\eta|^{\alpha}}|A|\left|\partial_{\xi} \phi\left(B \xi+f_{r}^{1}(\xi, \eta), C \eta+f_{r}^{2}(\xi, \eta)\right)\left(B+\partial_{\xi} f_{r}^{1}(\xi, \eta)\right)\right|+ \\
\frac{1}{|\eta|^{\alpha}}|A|\left|\partial_{\eta} \phi\left(B \xi+f_{r}^{1}(\xi, \eta), C \eta+f_{r}^{2}(\xi, \eta)\right) \partial_{\xi} f_{r}^{2}(\xi, \eta)\right|
\end{gathered}
$$

Below we estimate (3.6).

$$
\begin{gathered}
\frac{1}{|\eta|^{\alpha}}|A|\left|\partial_{\xi} \phi\left(B \xi+f_{r}^{1}(\xi, \eta), C \eta+f_{r}^{2}(\xi, \eta)\right)\left(B+\partial_{\xi} f_{r}^{1}(\xi, \eta)\right)\right| \leq \\
\frac{1}{|\eta|^{\alpha}}|A|\|\phi\|_{\alpha}\left|C \eta+f_{r}^{2}(\xi, \eta)\right|^{\alpha}\left|B+\partial_{\xi} f_{r}^{1}(\xi, \eta)\right| \leq|A|\|\phi\|_{\alpha}\left[\frac{\left|C \eta+f_{r}^{2}(\xi, \eta)\right|}{|\eta|}\right]^{\alpha}\left|B+\partial_{\xi} f_{r}^{1}(\xi, \eta)\right| \leq
\end{gathered}
$$




$$
\begin{gathered}
|A|\|\phi\|_{\alpha}\left[|C|+\frac{\left|f_{r}^{2}(\xi, \eta)\right|}{|\eta|}\right]^{\alpha}\left[|B|+O\left(r^{\alpha}\right)\right] \leq|A|\|\phi\|_{\alpha}\left[|C|+\frac{\left|\int_{0}^{1} \frac{d}{d t} f_{r}^{2}(\xi, t \eta) d t\right|}{|\eta|}\right]^{\alpha}\left[|B|+O\left(r^{\alpha}\right)\right] \leq \\
|A|\|\phi\|_{\alpha}\left[|C|+\frac{\left|\int_{0}^{1} \partial_{\eta} f_{r}^{2}(\xi, t \eta) d t\right||\eta|}{|\eta|}\right]^{\alpha}\left[|B|+O\left(r^{\alpha}\right)\right] \leq|A|\|\phi\|_{\alpha}\left[|C|+O\left(r^{\alpha}\right)\right]^{\alpha}\left[|B|+O\left(r^{\alpha}\right)\right] \leq \\
{\left[|A||B \| C|^{\alpha}+O\left(r^{\alpha}\right)\right]\|\phi\|_{\alpha}}
\end{gathered}
$$

Now we estimate (3.7).

$$
\begin{gathered}
\frac{1}{|\eta|^{\alpha}}|A|\left|\partial_{\eta} \phi\left(B \xi+f_{r}^{1}(\xi, \eta), C \eta+f_{r}^{2}(\xi, \eta)\right) \partial_{\xi} f_{r}^{2}(\xi, \eta)\right| \leq \\
|A|\|\phi\|_{\alpha}\left|B \xi+f_{r}^{1}(\xi, \eta)\right|^{\alpha} \frac{\left|\partial_{\xi} f_{r}^{2}(\xi, \eta)\right| \mid}{|\eta|^{\alpha}} \leq O\left(r^{\alpha}\right)\|\phi\|_{\alpha}
\end{gathered}
$$

The last inequality follows by considering the cases $|\xi| \leq r,|\xi|>r$.

We conclude that, $\frac{\left|\partial_{\xi} T(\phi)\right|}{|\eta|^{\alpha}} \leq\left[|A||B||C|^{\alpha}+O\left(r^{\alpha}\right)\right]\|\phi\|_{\alpha}$.

Since there is a symmetry on the regularity assumptions with respect to the variables $\xi$ and $\eta$, a similar calculation to the previous one would give:

$$
\frac{\left|\partial_{\eta} T(\phi)\right|}{|\xi|^{\alpha}} \leq\left[|A||B|^{\alpha}|C|+O\left(r^{\alpha}\right)\right]\|\phi\|_{\alpha}
$$

Since $|A||B||C|<1$, if $\alpha$ is sufficiently close to 1 , the two previous inequalities imply that $\mathcal{E}$ is invariant under $T$ and $T$ is a contraction.

Theorem 5. Let $X$ be a Banach space and $L \in \mathcal{L}(X)$. Suppose $|\sigma(L)| \subset(a, b) \cup(c, d)$, where $0<a<b<1<c<d$. Let $S$ and $U$, respectively, the stable and unstable subspaces of $L$. Let $F(x)=: L x+f(x)$ be a local mapping, where $f(0)=0, f^{\prime}(0)=0$ and $f \in \mathcal{C}^{1, \alpha}$, locally where $0<\alpha \leq 1$. Then there exists a local function $R=: I+\phi$, where $\phi \in \mathcal{C}^{1, \alpha}$ also locally, $\phi(0)=0, \phi^{\prime}(0)=0$, such that $S$ and $U$ are invariant under $G=: R F R^{-1}$ and $G(x)=: L x+g(x)$, where $g(0)=0, g^{\prime}(0)=0, g \in \mathcal{C}^{1, \alpha}$ locally.

Proof: We consider the following change of variables:

$$
R:\left\{\begin{array}{l}
x=: \xi-\gamma_{u}(\eta) \\
y=: \eta-\gamma_{s}(\xi)
\end{array}\right.
$$

where $\gamma_{s}, \gamma_{u}$ are given by Lemma 4 . 
From the Inverse Function Theorem it follows that $R^{-1}$ has the form:

$$
R^{-1}:\left\{\begin{array}{l}
\xi=x+d(x, y) \\
\eta=y+e(x, y)
\end{array}\right.
$$

where $d(0,0)=0, d^{\prime}(0,0)=0, e(0,0)=0, e^{\prime}(0,0)=0$.

Let us show now that the functions $d$ and $e$ belong to the class $\mathcal{C}^{1, \alpha}$ locally. From the expressions of $R$ and $R^{-1}$ it follows that,

$$
x=x+d(x, y)-\gamma_{u}(y+e(x, y)) \text { and so, } d(x, y)=\gamma_{u}(y+e(x, y)) .
$$

Similarly, $e(x, y)=\xi_{s}(x+d(x, y))$.

Therefore,

$$
\begin{aligned}
& \partial_{x} d(x, y)=\gamma_{u}^{\prime}(y+e(x, y)) \partial_{x} e(x, y), \quad \partial_{x} e(x, y)=\gamma_{s}^{\prime}(x+e(x, y))\left[I+\partial_{x} d(x, y)\right] \\
& \frac{\left|\partial_{x} d\left(x_{1}, y_{1}\right)-\partial_{x} d\left(x_{2}, y_{2}\right)\right|}{\left(\left|x_{1}-x_{2}\right|+\left|y_{1}-y_{2}\right|\right)^{\alpha}}=\frac{\left|\gamma_{u}^{\prime}\left(y_{1}+e\left(x_{1}, y_{1}\right)\right) \partial_{x} e\left(x_{1}, y_{1}\right)-\gamma_{u}^{\prime}\left(y_{2}+e\left(x_{2}, y_{2}\right)\right) \partial_{x} e\left(x_{2}, y_{2}\right)\right|}{\left(\left|x_{1}-x_{2}\right|+\left|y_{1}-y_{2}\right|\right)^{\alpha}}= \\
& \frac{\mid\left[\gamma_{u}^{\prime}\left(y_{1}+e\left(x_{1}, y_{1}\right)\right)-\gamma_{u}^{\prime}\left(y_{2}+e\left(x_{2}, y_{2}\right)\right)\right] \partial_{x} e\left(x_{1}, y_{1}\right)+\gamma_{u}^{\prime}\left(y_{2}+e\left(x_{2}, y_{2}\right)\right)\left[\partial_{x} e\left(x_{1}, y_{1}\right)-\partial_{x} e\left(x_{2}, y_{2}\right) \mid\right.}{\left(\left|x_{1}-x_{2}\right|+\left|y_{1}-y_{2}\right|\right)^{\alpha}} \\
& \leq \frac{\left|\gamma_{u}^{\prime}\left(y_{1}+e\left(x_{1}, y_{1}\right)\right)-\gamma_{u}^{\prime}\left(y_{2}+e\left(x_{2}, y_{2}\right)\right)\right|\left|\partial_{x} e\left(x_{1}, y_{1}\right)\right|}{\left(\left|x_{1}-x_{2}\right|+\left|y_{1}-y_{2}\right|\right)^{\alpha}}+\frac{\mid \gamma_{u}^{\prime}\left(y_{2}+e\left(x_{2}, y_{2}\right)|| \partial_{x} e\left(x_{1}, y_{1}\right)-\partial_{x} e\left(x_{2}, y_{2}\right) \mid\right.}{\left(\left|x_{1}-x_{2}\right|+\left|y_{1}-y_{2}\right|\right)^{\alpha}} \\
& \leq \text { Const. }\left|\partial_{x} e\left(x_{1}, y_{1}\right)\right| \frac{\left|y_{1}+e\left(x_{1}, y_{1}\right)-y_{2}-e\left(x_{2}, y_{2}\right)\right|^{\alpha}}{\left(\left|x_{1}-x_{2}\right|+\left|y_{1}-y_{2}\right|\right)^{\alpha}}+\mid \gamma_{u}^{\prime}\left(y_{2}+e\left(x_{2}, y_{2}\right) \mid \frac{\left|\partial_{x} e\left(x_{1}, y_{1}\right)-\partial_{x} e\left(x_{2}, y_{2}\right)\right|}{\left(\left|x_{1}-x_{2}\right|+\left|y_{1}-y_{2}\right|\right)^{\alpha}} \leq\right. \\
& \text { Const. } \mid \frac{\left|y_{1}-y_{2}\right|^{\alpha}+\left|e\left(x_{1}, y_{1}\right)-e\left(x_{2}, y_{2}\right)\right|^{\alpha}}{\left(\left|x_{1}-x_{2}\right|+\left|y_{1}-y_{2}\right|\right)^{\alpha}}+O\left(\left[\left|y_{2}\right|+\left|e\left(x_{2}, y_{2}\right)\right|\right]^{\alpha}\right) \frac{\left|\partial_{x} e\left(x_{1}, y_{1}\right)-\partial_{x} e\left(x_{2}, y_{2}\right)\right|}{\left(\left|x_{1}-x_{2}\right|+\left|y_{1}-y_{2}\right|\right)^{\alpha}} \leq \\
& \text { Const. }+ \text { Const. } \mid \frac{\left|\int_{0}^{1} \frac{d}{d t} e\left(t x_{1}+(1-t) x_{2}, t y_{1}+(1-t) y_{2}\right) d t\right|^{\alpha}}{\left(\left|x_{1}-x_{2}\right|+\left|y_{1}-y_{2}\right|\right)^{\alpha}}+ \\
& O\left(\left[\left|y_{2}\right|+\left|e\left(x_{2}, y_{2}\right)\right|\right]^{\alpha}\right) \frac{\left|\partial_{x} e\left(x_{1}, y_{1}\right)-\partial_{x} e\left(x_{2}, y_{2}\right)\right|}{\left(\left|x_{1}-x_{2}\right|+\left|y_{1}-y_{2}\right|\right)^{\alpha}} \leq \\
& \text { Const. } \frac{\left[\int_{0}^{1}\left|\partial_{x} e\left(t x_{1}+(1-t) x_{2}, t y_{1}+(1-t) y_{2}\right)\right| d t\left|x_{1}-x_{2}\right|+\int_{0}^{1}\left|\partial_{y} e\left(t x_{1}+(1-t) x_{2}, t y_{1}+(1-t) y_{2}\right)\right| d t\left|y_{1}-y_{2}\right|\right]^{\alpha}}{\left(\left|x_{1}-x_{2}\right|+\left|y_{1}-y_{2}\right|\right)^{\alpha}} \\
& + \text { Const. }+O\left(\left[\left|x_{2}\right|+\left|y_{2}\right|\right]^{\alpha}\right) \frac{\left|\partial_{x} e\left(x_{1}, y_{1}\right)-\partial_{x} e\left(x_{2}, y_{2}\right)\right|}{\left(\left|x_{1}-x_{2}\right|+\left|y_{1}-y_{2}\right|\right)^{\alpha}} \leq
\end{aligned}
$$




$$
\begin{gathered}
\text { Const. } \frac{\left[\left|x_{1}-x_{2}\right|+\left|y_{1}-y_{2}\right|\right]^{\alpha}}{\left(\left|x_{1}-x_{2}\right|+\left|y_{1}-y_{2}\right|\right)^{\alpha}}+\text { Const. }+O\left(\left[\left|x_{2}\right|+\left|y_{2}\right|\right]^{\alpha}\right) \frac{\left|\partial_{x} e\left(x_{1}, y_{1}\right)-\partial_{x} e\left(x_{2}, y_{2}\right)\right|}{\left(\left|x_{1}-x_{2}\right|+\left|y_{1}-y_{2}\right|\right)^{\alpha}} \leq \\
\text { Const. }+O\left(\left[\left|x_{2}\right|+\left|y_{2}\right|\right]^{\alpha}\right) \frac{\left|\partial_{x} e\left(x_{1}, y_{1}\right)-\partial_{x} e\left(x_{2}, y_{2}\right)\right|}{\left(\left|x_{1}-x_{2}\right|+\left|y_{1}-y_{2}\right|\right)^{\alpha}}
\end{gathered}
$$

We conclude that,

$$
\frac{\left|\partial_{x} d\left(x_{1}, y_{1}\right)-\partial_{x} d\left(x_{2}, y_{2}\right)\right|}{\left(\left|x_{1}-x_{2}\right|+\left|y_{1}-y_{2}\right|\right)^{\alpha}} \leq \text { Const. }+O\left(\left[\left|x_{2}\right|+\left|y_{2}\right|\right]^{\alpha}\right) \frac{\left|\partial_{x} e\left(x_{1}, y_{1}\right)-\partial_{x} e\left(x_{2}, y_{2}\right)\right|}{\left(\left|x_{1}-x_{2}\right|+\left|y_{1}-y_{2}\right|\right)^{\alpha}}
$$

Now we will use a similar approach for $e(x, y)$, to obtain: Therefore,

$$
\frac{\left|\partial_{x} e\left(x_{1}, y_{1}\right)-\partial_{x} e\left(x_{2}, y_{2}\right)\right|}{\left(\left|x_{1}-x_{2}\right|+\left|y_{1}-y_{2}\right|\right)^{\alpha}} \leq \text { Const. }+O\left(\left[\left|x_{2}\right|+\left|y_{2}\right|\right]^{\alpha}\right) \frac{\left|\partial_{x} d\left(x_{1}, y_{1}\right)-\partial_{x} d\left(x_{2}, y_{2}\right)\right|}{\left(\left|x_{1}-x_{2}\right|+\left|y_{1}-y_{2}\right|\right)^{\alpha}}
$$

The two previous inequalities imply that $\partial_{x} d$ and $\partial_{x} e$ belong to the class $\mathcal{C}^{0, \alpha}$ in a sufficiently small neighborhood of the origin. Since similar results can be proved for $\partial_{y} d$ and $\partial_{y} e$, we conclude that $d$ and $e$ belong to the class $\mathcal{C}^{1, \alpha}$ in a sufficiently small neighborhood of the origin.

We define

$$
G(x, y)=\left(\begin{array}{l}
G_{s}(x, y) \\
G_{u}(x, y)
\end{array}\right)=\left(\begin{array}{l}
G_{s}\left(\xi-\gamma_{u}(\eta), \eta-\gamma_{s}(\xi)\right) \\
G_{u}\left(\xi-\gamma_{u}(\eta), \eta-\gamma_{s}(\xi)\right)
\end{array}\right)=:\left(\begin{array}{c}
F_{s}(\xi, \eta)-\gamma_{u}\left(F_{u}(\xi, \eta)\right) \\
F_{u}(\xi, \eta)-\gamma_{s}\left(F_{s}(\xi, \eta)\right),
\end{array}\right)
$$

where $\xi=x+d(x, y)$ and $\eta=y+e(x, y)$ and $\gamma_{s}, \gamma_{u}$ are given by Lemma 4 .

In terms of the new variables we have $x=0 \Leftrightarrow \xi=\gamma_{u}(\eta)$ and $y=0 \Leftrightarrow \eta=\gamma_{s}(\xi)$.

Therefore $G_{u}(x, 0)=F_{u}\left(\xi, \gamma_{1}(\xi)\right)-\gamma_{1}\left(F_{s}\left(\xi, \gamma_{1}(\xi)\right)\right) \equiv 0$.

Also $G_{s}(0, y) \equiv 0$.

If we recall the expressions of $F_{s}$ and $F_{u}$ a simple calculation shows that,

$$
G(x, y)=\left(\begin{array}{l}
G_{s}(x, y) \\
G_{u}(x, y)
\end{array}\right)=\left(\begin{array}{c}
L_{s} x+g_{s}(x, y) \\
L_{u} y+g_{u}(x, y)
\end{array}\right), g(x, y)=:\left(\begin{array}{c}
g_{s}(x, y) \\
g_{u}(x, y)
\end{array}\right)
$$

where $g$ belongs to the class $\mathcal{C}^{1, \alpha}, g_{u}(x, 0) \equiv 0$ and $g_{s}(0, y) \equiv 0$. 


\section{Proof of the Main Theorem.}

The proof of our main theorem follows easily from the next result:

Theorem 6. Under the assumptions of Theorem 2, if $x=(\xi, \eta)$ and $F(x)$ be given by:

$$
F(x)=:\left(\begin{array}{c}
L_{s} \xi+f_{s}(\xi, \eta) \\
L_{u} \eta+f_{u}(\xi, \eta)
\end{array}\right)
$$

where $f(0,0)=0, f^{\prime}(0)=0, f_{s}(0, \eta) \equiv 0, f_{u}(\xi, 0) \equiv 0$.

Then $F$ is locally conjugate to $L$.

Proof: We first extend $f$ to the whole space as in (2.1) by considering $f_{r}(\xi, \eta)=: \Phi_{r}(\xi / r, \eta / r) f(\xi, \eta)$, where $r$ is sufficiently small. To simplify the notation we will use $\mathrm{f}$ in the place of $f_{r}$ in the following analysis.

In order to find the conjugation $h$ we will show that the equation: $T(h)=-f$ has a solution $h$ in an appropriate space, where $T(h)=: h(F(x))-\operatorname{Lh}(x)$.

Following [1], we consider the space $\mathcal{L}=:\left\{h \in C^{1}(X, X): h(0)=0, h^{\prime}(0)=0\right\}$ and the following subspaces: $\mathcal{L}_{1}=:\left\{h \in \mathcal{L}: \partial_{\eta} h=0\right\}, \mathcal{L}_{2}=:\left\{h \in \mathcal{L}: \partial_{\xi} h=0\right\}$, $\mathcal{L}_{3}=:\{h \in \mathcal{L}: h(0, \eta)=h(\xi, 0)=0\}$.

We consider the following projections $P_{i}: \mathcal{L} \rightarrow \mathcal{L}_{i}, i=1,2,3$ :

$$
\left(P_{1} h\right)(x)=: h(\xi, 0),\left(P_{2} h\right)(x)=: h(0, \eta),\left(P_{3} h\right)(x)=: h(\xi, \eta)-h(\xi, 0)-h(0, \eta)
$$

Let $h=h^{1}+h^{2}+h^{3}$, where $h^{i} \in \mathcal{L}_{i}, i=1,2,3$.

First we point out that the subspaces $\mathcal{L}_{3}, \mathcal{L}_{1}+\mathcal{L}_{3}, \quad \mathcal{L}_{2}+\mathcal{L}_{3}$ are invariant under $T$ and $T$ is linear. This implies that solving the equation $T(h)=-f$ is equivalent to solving the system of equations:

$$
\begin{gathered}
P_{1} T\left(h^{1}\right)=P_{1} T\left(h^{1}+h^{2}+h^{3}\right)=-P_{1} f \\
P_{2} T\left(h^{2}\right)=P_{2} T\left(h^{1}+h^{2}+h^{3}\right)=-P_{2} f \\
P_{3} T\left(h^{1}+h^{2}+h^{3}\right)=-P_{3} f
\end{gathered}
$$


The first equation depends only on $\xi$ and the second one depends only on $\eta$. Therefore they can be solved separated. Once we had solved the two first equations, we substitute in the third to obtain:

$$
P_{3} T\left(h^{3}\right)=-P_{3}\left(f+T\left(h^{1}+h^{2}\right)\right)
$$

Our assumptions imply that:

$$
\begin{gathered}
\left(P_{1} f\right)(\xi, \eta)=f(\xi, 0)=\left(\begin{array}{c}
f_{s}(\xi, 0) \\
f_{u}(\xi, 0)
\end{array}\right)=\left(\begin{array}{c}
f_{s}(\xi, 0) \\
0
\end{array}\right) \\
P_{1} T\left(h^{1}\right)(x)=h^{1}(F(\xi, 0))-L h^{1}(\xi, 0)=\left(\begin{array}{c}
h_{s}^{1}\left(F_{s}(\xi, 0), F_{u}(\xi, 0)\right)-L_{s} h_{s}^{1}(\xi, 0) \\
h_{u}^{1}\left(F_{s}(\xi, 0), F_{u}(\xi, 0)\right)-L_{u} h_{u}^{1}(\xi, 0)
\end{array}\right)= \\
\left(\begin{array}{c}
h_{s}^{1}\left(F_{s}(\xi, 0), 0\right)-L_{s} h_{s}^{1}(\xi, 0) \\
h_{u}^{1}\left(F_{s}(\xi, 0), 0\right)-L_{u} h_{u}^{1}(\xi, 0)
\end{array}\right)
\end{gathered}
$$

Therefore equation (4.1) will be equivalent to:

$$
\left(\begin{array}{c}
h_{s}^{1}\left(F_{s}(\xi, 0), 0\right)-L_{s} h_{s}^{1}(\xi, 0) \\
h_{u}^{1}\left(F_{s}(\xi, 0), 0\right)-L_{u} h_{u}^{1}(\xi, 0)
\end{array}\right)=\left(\begin{array}{c}
f_{s}(\xi, 0) \\
0
\end{array}\right)
$$

We see that $h_{u}^{1}=0$, solves the equation that appears in the second component of the above equation. The first component gives:

$$
h_{s}^{1}\left(F_{s}(\xi, 0), 0\right)-L_{s} h_{s}^{1}(\xi, 0)=f_{s}(\xi, 0)
$$

In a similar way we obtain solving equation (4.2) is equivalent to:

$$
h_{u}^{2}\left(0, F_{u}(0, \eta)\right)-L_{u} h_{u}^{2}(0, \eta)=f_{u}(0, \eta)
$$

Each one of these two equations depends only in one variable and so, they can be solved independently.

If we let $\bar{F}(\xi)=: F_{s}(\xi, 0)=L_{s} \phi+f_{s}(\xi, 0)=: L_{s} \phi+\bar{f}(\xi)$ and $\phi(\xi)=: h_{s}^{1}(\xi, 0)$, (4.5) will be equivalent to:

$$
\phi(\xi)=L_{s}^{-1} \phi(\bar{F}(\xi))-L_{s}^{-1} \bar{f}(\xi)
$$


From Lemma 3 it follows that we can find a solution, $\phi$, in the space $\mathcal{C}^{1, \alpha}(\mathbb{X}, \mathbb{X})$, such that $\phi(0)=0, \phi^{\prime}(0)=0$, because $\left\|L_{s}^{-1}\right\|\left\|L_{s}\right\|^{1+\alpha}<\frac{\left(s_{+}\right)^{1+\alpha}}{s_{-}}<1$, if $\alpha$ is taken sufficiently close to 1. This follows from (1.3).

In a similar way we can solve equation (4.6), but we have to work with the inverse function.

If we let $\tilde{F}(\eta)=: F_{u}(0, \eta)=L_{u} \eta+f_{u}(0, \eta)=: L_{u} \phi+\tilde{f}(\eta)$ and $\psi(\xi)=: h_{u}^{1}(0, \eta),(4.6)$ will be equivalent to:

$$
L_{u} \psi(\tilde{F}(\eta))-L_{u} \psi(\eta)=\tilde{f}(\eta)
$$

Now we let $v=: \tilde{F}(\eta), G=: \tilde{F}^{-1}$ the above equation will be equivalent to:

$$
\psi(v)=L_{u} \psi(G(v))+\tilde{f}(G(v))
$$

Using the Inverse Theorem one can prove that $G(v)=: L_{u}^{-1} v+g(v)$ and so $g$ inherits some properties of $\tilde{f}=f(0, \cdot)$, for example $g(0)=0, g(v) \equiv 0$, for $v$ outside a ball of radius of $O(r)$. Since,

$$
\begin{gathered}
g^{\prime}=G^{\prime}-L_{u}^{-1}=\left(\tilde{F}^{-1}\right)^{\prime}-L_{u}^{-1}=\left(\tilde{F}^{\prime}\right)^{-1}-L_{u}^{-1}=\left(\tilde{F}^{\prime}\right)^{-1}\left(L_{u}-\tilde{F}^{\prime}\right) L_{u}^{-1}= \\
-\left(L_{u}+\tilde{f}^{\prime}\right)^{-1}\left(\tilde{f}^{\prime}\right) L_{u}^{-1}=-L_{u}^{-1}\left(I+L_{u}^{-1} \tilde{f}^{\prime}\right)^{-1}\left(\tilde{f}^{\prime}\right) L_{u}^{-1}
\end{gathered}
$$

one can prove that $g^{\prime}(0)=0, g \in \mathcal{C}^{1, \alpha}(\mathbb{U}, \mathbb{U})$. Also $d(v)=: \tilde{f}(G(v))$ satisfies $d(0)=0, d^{\prime}(0)=$ $0, d \in \mathcal{C}^{1, \alpha}(\mathbb{U}, \mathbb{U})$.

Therefore equation (4.9) will be equivalent to:

$$
\psi(v)=L_{u} \psi\left(L_{u}^{-1} v+g(v)\right)+d(v)
$$

Using the fact that $\left\|L_{u}\right\|\left\|L_{u}^{-1}\right\|^{1+\alpha}<u^{+}\left(\frac{1}{\left(u_{-}\right)}\right)^{1+\alpha}<1$, if $\alpha$ is taken sufficiently close to 1 , by Lemma 3 , one can prove that (4.10) has a solution $\psi \in \mathcal{C}^{1, \alpha}(\mathbb{U}, \mathbb{U})$, satisfying $\psi(0)=0, \psi^{\prime}(0)=0$.

Let us analyze now equation (4.4):

$$
P_{3} T\left(h_{3}\right)=-P_{3}\left(f+T\left(h_{1}+h_{2}\right)\right)
$$

The problem of solving (4.11) has been reduced to solve in $\mathcal{L}_{3}$ an equation of the form:

$$
h(F(\xi, \eta))-L h(\xi, \eta)=\theta(\xi, \eta)
$$


where $\theta \in \mathcal{L}_{3}$.

Equation (4.11) is equivalent to the system:

$$
\begin{gathered}
h_{s}(F(\xi, \eta))-L_{s} h_{s}(\xi, \eta)=\theta_{s}(\xi, \eta) \\
h_{u}(F(\xi, \eta))-L_{u} h_{u}(\xi, \eta)=\theta_{u}(\xi, \eta)
\end{gathered}
$$

Equation (4.13) is equivalent to:

$$
\phi(\xi, \eta)=L_{u}^{-1} \phi\left(L_{s} \xi+f_{s}(\xi, \eta), L_{u} \eta+f_{u}(\xi, \eta)\right)-L_{u}^{-1} \gamma(\xi, \eta)
$$

where $\phi, \gamma \in \mathcal{L}_{3}^{\prime}=:\left\{h \in C^{1}(\mathbb{S} \times \mathbb{U}, \mathbb{U}): h(0, \eta)=h(\xi, 0)=0\right\}$.

In this space we consider the norm $\|\cdot\|_{\alpha}$ given in (2.6).

From (1.3) it follows that the operator: $\mathcal{T}(\phi)=L_{u}^{-1} \phi\left(L_{s} \xi+f_{s}(\xi, \eta), L_{u} \eta+f_{u}(\xi, \eta)\right)$ is a contraction in this space because

$$
\left|L_{u}^{-1}\right|\left|L_{s}\right|\left|L_{u}\right|^{\alpha} \leq \frac{s_{+} u_{+}^{\alpha}}{s_{-}}<1,\left.\left|L_{u}^{-1}\right| L_{s}\right|^{\alpha}\left|L_{u}\right| \leq \frac{u_{+} s_{+}^{\alpha}}{s_{-}}<1
$$

if we take $\alpha$ sufficiently close to 1 and $r$ is sufficiently close to 0 .

It follows from Lemma 5 that equation (4.14) has a solution $\phi \in \mathcal{L}_{3}^{\prime}$.

To solve equation (4.12) we have to use the inverse function $F^{-1}$.

If we let $(x, y)=: F(\xi, \eta)$ and $h_{s}=: \phi$, solving equation 4.12 will be equivalent to solve the equation:

$$
\phi(x, y)=L_{s} \phi\left(F_{s}^{-1}(x, y)\right)+\theta_{s}\left(F^{-1}(x, y)\right)
$$

If we let $F^{-1}(x, y)=:\left(L_{s}^{-1} x+\tilde{f}_{s}(x, y), L_{u}^{-1} u+\tilde{f}_{u}(x, y)\right), \theta_{s}\left(F^{-1}(x, y)\right)=: \tilde{\theta}(x, y)$, equation (4.15) will be equivalent to:

$$
\phi(x, y)=L_{s} \phi\left(L_{s}^{-1} x+\tilde{f}_{s}(x, y), L_{u}^{-1} y+\tilde{f}_{u}(x, y)\right)+\tilde{\theta}(x, y),
$$

where $\tilde{f}_{s}(0, y) \equiv 0, \tilde{f}_{u}(x, 0) \equiv 0, \tilde{\theta}(0, y) \equiv 0, \tilde{\theta}(x, 0) \equiv 0, \tilde{f}_{s}, \tilde{f}_{u} \in \mathcal{C}^{1, \alpha}$.

To prove that $\tilde{f}_{s}, \tilde{f}_{u} \in \mathcal{C}^{1, \alpha}$ we can use some ideas of the proof of Theorem 5

From (1.3) and Lemma 5, using an space similar to $\mathcal{L}_{3}^{\prime}$, one can prove that the operator:

$$
(T \phi)(x, y)=: L_{s} \phi\left(F^{-1}(x, y)\right)
$$


is a contraction because

$$
\left|L_{s}\right|\left|L_{s}^{-1}\right|\left|L_{u}^{-1}\right|^{\alpha} \leq \frac{s_{+}}{s_{-} u_{-}^{\alpha}}<1,\left|L_{s}\right|\left|L_{u}^{-1}\right|\left|L_{s}^{-1}\right|^{\alpha} \leq \frac{s_{+}}{u_{-} s_{-}^{\alpha}}<1
$$

if $\alpha$ is chosen sufficiently close to 1 and $r$ is sufficiently close to 0 .

From Lemma 5 it follows that we can find a solution $\phi$ of equation (4.15).

\section{Applications.}

Following [4] we consider the next abstract equation, that represents the functional formulation of many damped wave equations:

$$
\ddot{u}-2 \gamma \dot{u}+\mathcal{A} u=0 .
$$

It is defined in a Hilbert Space $E, \gamma$ is a negative real number and $\mathcal{A}$ is a self-adjoint positive operator on $E$,

$$
0<\mu=: \inf _{u \in \mathcal{D}(\mathcal{A})} \frac{\langle\mathcal{A} u, u\rangle}{\langle u, u\rangle}
$$

where $\langle\cdot, \cdot\rangle$ denotes the inner product of $E$ and $\mathcal{D}(\mathcal{A})$ indicates the domain of $\mathcal{A}$. As it is well know, $\mu$ coincides with the smallest element of the spectrum of $\mathcal{A}$.

Remark 2. The change of variables $v(t)=: u(-t)$ transform (5.1) in

$$
\ddot{v}+2 \gamma \dot{v}+\mathcal{A} v=0 .
$$

In the following, $E^{1 / 2}$ will denote the Hilbert space consisting of the domain of the operator $(\mathcal{A}+\xi I)^{1 / 2}$ endowed with the inner product:

$$
\langle u, \hat{u}\rangle_{1 / 2}=:\left\langle(\mathcal{A}+\xi I)^{1 / 2} u,(\mathcal{A}+\xi I)^{1 / 2} \hat{u}\right\rangle
$$

where $\xi$ is a real number $\xi>-\mu$. Different choices of $\xi$ result in the same vector space with different but equivalent inner products. Define $\mathbb{E}=: E^{1 / 2} \times E$.

Equation (5.1) is equivalent to the system:

$$
\dot{U}=\gamma U+\mathbb{A} U
$$


in $\mathbb{E}$, where

$$
\mathbb{E} \ni U=\left(\begin{array}{l}
u \\
w
\end{array}\right)=:\left(\begin{array}{c}
u \\
-\gamma u+\dot{u}
\end{array}\right), \text { and } \mathbb{A} U=\mathbb{A}\left(\begin{array}{l}
u \\
w
\end{array}\right)=:\left(\begin{array}{c}
w \\
\gamma^{2} u-\mathcal{A} u
\end{array}\right)
$$

and it is known that it defines a $C^{0}$-group $T(t)=: e^{\gamma t} e^{\mathbb{A} t}$.

We consider the following inner product in $\mathbb{E}$, where we choose different values of $\xi$, according to the values of $\gamma^{2}$,

$$
\langle U, \hat{U}\rangle_{\mathbb{E}}=:\left\{\begin{array}{lc}
\left\langle\left(A-\gamma^{2} I\right)^{1 / 2} u,\left(A-\gamma^{2} I\right)^{1 / 2} \hat{u}\right\rangle+\langle w, \hat{w}\rangle, & \text { if } \gamma^{2}<\mu, \\
\left\langle\left(A-\left(\varepsilon^{2}-\mu\right) I\right)^{1 / 2} u,\left(A-\left(\varepsilon^{2}-\mu\right) I\right)^{1 / 2} \hat{u}\right\rangle+\langle w, \hat{w}\rangle, & \text { if } \gamma^{2}=\mu \\
\left\langle\left(A-\left(\gamma^{2}-2 \mu\right) I\right)^{1 / 2} u,\left(A-\left(\gamma^{2}-2 \mu\right) I\right)^{1 / 2} \hat{u}\right\rangle+\langle w, \hat{w}\rangle, & \text { if } \gamma^{2}>\mu .
\end{array}\right.
$$

where $\varepsilon>0$ is a small number to be chosen later on.

If $T(t)$ denotes the group generated by $\gamma I+\mathbb{A}$, from [4] it follows that the following estimates hold:

$$
\left\{\begin{array}{l}
\text { if } \gamma^{2}<\mu,|T(t)| \leq e^{\gamma t}, \text { for all } t \text { in } \mathbb{R} \\
\text { if } \gamma^{2}=\mu,|T(t)| \leq e^{\gamma t+\varepsilon|t|}, \text { for all } t \text { in } \mathbb{R} \\
\text { if } \gamma^{2}>\mu,|T(t)| \leq e^{\left[\gamma t-\sqrt{\gamma^{2}-\mu}|t|\right], \text { for all } t \text { in } \mathbb{R}}
\end{array}\right.
$$

Consider now the system of equations:

$$
\left\{\begin{array}{l}
\ddot{u}-2 \gamma_{s} \dot{u}+\mathcal{A} u=0 \\
\ddot{v}-2 \gamma_{u} \dot{v}+\mathcal{A} v=0
\end{array}\right.
$$

where $\gamma_{s}<0$ and $\gamma_{u}>0$.

As in equation (5.4), system (5.7) is equivalent to:

$$
\left\{\begin{array}{l}
\dot{U}=\gamma_{s} U+\mathbb{A}_{s} U \\
\dot{V}=\gamma_{u} V+\mathbb{A}_{u} V
\end{array}\right.
$$

in $\mathbb{E} \times \mathbb{E}$, where

$$
\begin{aligned}
& \mathbb{E} \ni U=\left(\begin{array}{c}
u \\
w_{1}
\end{array}\right)=:\left(\begin{array}{c}
u \\
-\gamma_{s} u+\dot{u}
\end{array}\right) \text {, and } \mathbb{A}_{s} U=\mathbb{A}_{s}\left(\begin{array}{c}
u \\
w_{1}
\end{array}\right)=:\left(\begin{array}{c}
w_{1} \\
\gamma_{s}^{2} u-\mathcal{A} u
\end{array}\right) \\
& \mathbb{E} \ni V=\left(\begin{array}{c}
v \\
w_{2}
\end{array}\right)=:\left(\begin{array}{c}
v \\
-\gamma_{u} v-\dot{v}
\end{array}\right) \text {, and } \mathbb{A}_{u} U=\mathbb{A}_{u}\left(\begin{array}{c}
v \\
w_{2}
\end{array}\right)=:\left(\begin{array}{c}
w_{2} \\
\gamma_{u}{ }^{2} v-\mathcal{A} v
\end{array}\right)
\end{aligned}
$$


and it is known that it defines a $C^{0}$-group $\left(T_{s}(t), T_{u}(t)\right)=:\left(e^{\gamma_{s} t} e^{\mathbb{A} t}, e^{\gamma_{u} t} e^{\mathbb{A} t}\right)$.

From the above remarks, we obtain the following estimates for the semigroups $T_{s}(t), T_{u}(t)$.

$$
\begin{aligned}
& \left\{\begin{array}{l}
\text { if } \gamma_{s}^{2}<\mu,\left|T_{s}(t)\right| \leq e^{\gamma_{s} t}, \text { for all } t \text { in } \mathbb{R}, \\
\text { if } \gamma_{s}^{2}=\mu,\left|T_{s}(t)\right| \leq e^{\gamma_{s} t+\varepsilon|t|}, \text { for all } t \text { in } \mathbb{R}, \\
\text { if } \gamma_{s}^{2}>\mu,\left|T_{s}(t)\right| \leq e^{\left.\left[\gamma_{s} t-\sqrt{\gamma_{s}^{2}-\mu} \mid t\right]\right]}, \text { for all } t \text { in } \mathbb{R} .
\end{array}\right. \\
& \left\{\begin{array}{l}
\text { if } \gamma_{u}^{2}<\mu,\left|T_{u}(t)\right| \leq e^{\gamma_{u} t}, \text { for all } t \text { in } \mathbb{R}, \\
\text { if } \gamma_{u}^{2}=\mu,\left|T_{u}(t)\right| \leq e^{\gamma_{u} t+\varepsilon|t|}, \text { for all } t \text { in } \mathbb{R}, \\
\text { if } \gamma_{u}^{2}>\mu,\left|T_{u}(t)\right| \leq e^{\left[\gamma_{u} t-\sqrt{\gamma_{u}^{2}-\mu}|t|\right]}, \text { for all } t \text { in } \mathbb{R} .
\end{array}\right.
\end{aligned}
$$

Let us analyze all the possible cases, concerning the nonresonance conditions. We take $\varepsilon<\min \left\{-\frac{\gamma_{s}}{3}, \frac{\gamma_{u}}{3}\right\}$.

If $\gamma_{s}^{2} \leq \mu$ and $\gamma_{u}^{2} \leq \mu$ the nonresonance conditions will be automatically satisfied.

If $\gamma_{s}^{2}>\mu$ and $\gamma_{u}^{2} \leq \mu$ we must require that:

$$
2 \sqrt{\gamma_{s}^{2}-\mu}<-\gamma_{s}-\sqrt{\gamma_{s}^{2}-\mu} \text { (or equivalently } \gamma_{s}^{2}<\frac{9}{8} \mu \text { ) and } 2 \sqrt{\gamma_{s}^{2}-\mu}<\gamma_{u} \text {. }
$$

In analogy with the previous case, if $\gamma_{u}^{2}>\mu$ and $\gamma_{s}^{2} \leq \mu$ we must require that:

$$
2 \sqrt{\gamma_{u}^{2}-\mu}<\gamma_{u}-\sqrt{{\gamma_{u}}^{2}-\mu} \text { (or equivalently } \gamma_{u}^{2}<\frac{9}{8} \mu \text { ) and } 2 \sqrt{{\gamma_{u}}^{2}-\mu}<-\gamma_{s} .
$$

If $\gamma_{s}^{2}>\mu$ and $\gamma_{u}^{2}>\mu$ we must require that:

$$
\begin{gathered}
\left.2 \sqrt{\gamma_{s}^{2}-\mu}<-\gamma_{s}-\sqrt{\gamma_{s}^{2}-\mu} \text { (or equivalently } \gamma_{s}^{2}<\frac{9}{8} \mu\right) \\
\left.2 \sqrt{\gamma_{u}^{2}-\mu}<\gamma_{u}-\sqrt{\gamma_{u}^{2}-\mu} \text { (or equivalently } \gamma_{u}^{2}<\frac{9}{8} \mu\right) \\
2 \sqrt{\gamma_{s}^{2}-\mu}<\gamma_{u}-\sqrt{\gamma_{u}^{2}-\mu} \\
2 \sqrt{\gamma_{u}^{2}-\mu}<-\gamma_{s}-\sqrt{\gamma_{s}^{2}-\mu} .
\end{gathered}
$$

Consider now the nonlinear system of equations,

$$
\left\{\begin{array}{l}
\ddot{u}-2 \gamma_{s} \dot{u}+\mathcal{A} u=f_{1}(u, \dot{u}, v, \dot{v}) \\
\ddot{v}-2 \gamma_{u} \dot{v}+\mathcal{A} v=f_{2}(u, \dot{u}, v, \dot{v}),
\end{array}\right.
$$


As above, if we consider the change of variables,

$$
\begin{aligned}
& U=\left(\begin{array}{l}
u \\
w_{1}
\end{array}\right)=:\left(\begin{array}{c}
u \\
-\gamma_{s} u+\dot{u}
\end{array}\right) \\
& V=\left(\begin{array}{c}
v \\
w_{2}
\end{array}\right)=:\left(\begin{array}{c}
v \\
-\gamma_{u} v-\dot{v}
\end{array}\right)
\end{aligned}
$$

system (5.11) can be put in the form,

$$
\left\{\begin{array}{l}
\dot{U}=\gamma_{s} U+\mathbb{A}_{s} U+F_{1}(U, V) \\
\dot{V}=\gamma_{u} V+\mathbb{A}_{u} V+F_{2}(U, V)
\end{array}\right.
$$

$F_{1}(U, V)=:\left(\begin{array}{c}0 \\ f_{1}\left(u, w_{1}+\gamma_{s} u, v,-w_{2}-\gamma_{u} v\right)\end{array}\right), \quad F_{2}(U, V)=:\left(\begin{array}{c}0 \\ f_{2}\left(u, w_{1}+\gamma_{s} u, v,-w_{2}-\gamma_{u} v\right)\end{array}\right)$.

If $f_{1}(0)=0, f_{1}^{\prime}(0)=0 f_{2}(0)=0, f_{2}^{\prime}(0)=0, f_{1}, f_{2} \in \mathcal{C}^{1, \alpha}$ locally, for $\alpha$ sufficiently close to 1 and the above nonresonance conditions are satisfied, then our results imply that system (5.12) can be conjugated to the linear system:

$$
\left\{\begin{array}{l}
\dot{U}=\gamma_{s} U+\mathbb{A}_{s} U \\
\dot{V}=\gamma_{u} V+\mathbb{A}_{u} V
\end{array}\right.
$$

\section{REFERENCES}

[1] Aronson, S. Kh., Belitskii, G. R. and Zhuzhoma, E. V. (1996). Introduction to the Qualitative Theory of Dynamical Systems on Surfaces, Translations of Mathematical Monographs, A. M. S. , Volume 153, 268-277.

[2] Hartman, P. (1960). On local homeomorphisms of euclidean spaces, Boletin de la Sociedad Matematica Mexicana, 5, 2, 220-241.

[3] Henry, D.(1981). Evolution Equations in Banach Spaces, Lecture Notes, Instituto de Matemática e Estatística. Universidade de São Paulo.

[4] Mora, X. and J. Solà-Morales; J. (1987). Existence and Non-existence of Finite-dimensional Globally Attracting invariant Manifolds in Semilinear Damped Wave Equations, Dynamics of infinite-dimensional systems (Lisbon, 1986), 187-210, NATO Adv. Sci. Inst. Ser. F: Comput. Systems Sci., 37, Springer, Berlin-New York.

[5] Rodrigues, H. M., J. Solà-Morales,J. (2003). Linearization of Class $C^{1}$ for Contractions on Banach Spaces, Pre-print. 
[6] G. R. Sell, Smooth linearization near a fixed point, American J. of Math., 107, 1035-1091 (1985).

[7] S. Sternberg, Local contractions and a theorem of Poincaré, American J. of Math., 79, 809-824 (1957).

[8] B. Tan, Invariant Manifolds, invariant foliations and linearization theorems in Banach Space, PhD Thesis, Georgia Institute of Technology, (1998).

[9] S. van Strien, Smooth linearization of hyperbolic fixed points without resonance conditions, J. Differential Equations 85 No. 1, 66-90 (1990). 